\title{
On Pebble Automata for Data Languages with Decidable Emptiness Problem*
}

\author{
Tony Tan \\ School of Informatics \\ University of Edinburgh \\ Email: ttan@inf.ed.ac.uk
}

\begin{abstract}
In this paper we study a subclass of pebble automata (PA) for data languages for which the emptiness problem is decidable.

Namely, we introduce the so-called top view weak PA. Roughly speaking, top view weak PA are weak PA where the equality test is performed only between the data values seen by the two most recently placed pebbles. The emptiness problem for this model is decidable. We also show that it is robust: alternating, nondeterministic and deterministic top view weak PA have the same recognition power. Moreover, this model is strong enough to accept all data languages expressible in Linear Temporal Logic with the future-time operators, augmented with one register freeze quantifier.
\end{abstract}

\section{Introduction}

Regular languages are clearly one of the most important concepts in computer science. They have applications in basically all branches of computer science. It can be argued that the following properties contributed to their success.

1. Expressiveness: In many settings regular languages are powerful enough to capture the kinds of patterns that have to described.

*This work was done while the author was in the Department of Computer Science in Technion - Israel Institute of Technology. It can also be found as a technical report in [17]. 
2. Decidability: Unlike many general computational models, the mechanisms associated with regular languages allow one to perform automated semantic analysis.

3. Efficiency: The model checking problem, that is, testing whether a given string is accepted by a given automaton can be solved in polynomial time.

4. Closure properties: The regular languages possess all important closure properties.

5. Robustness: The class of regular languages has many characterizations. For example, various extensions like nondeterminism and alternation do not add any expressive power. Another characterizations include regular expressions, monoids and monadic second-order logic.

Moreover, similar notion of regularity has been successfully generalized to other kind of structures, including infinite strings and finite, as well as infinite, ranked or unranked, trees. Most recent applications of regular languages (on infinite strings and finite, unranked trees, respectively) are in model checking and XML processing.

- In model checking a system is a finite state one and properties are specified in a logic like LTL. Satisfiability of a formula in a system is checked on the structure that is the product of the system automaton and an automaton corresponding to the formula. The step from the "real" system to its finite state representation usually involves many abstraction, especially with respect to data values (variables, process numbers, etc.). Often their range is restricted to a finite domain.

Even though this approach has been successful and found its way into large scale industrial applications, the finite abstraction have some inherent shortcomings. As as example, $n$ identical processes with $m$ states each give rise to an overall model of size $m^{n}$. If the number of processes is unbounded or unknown in advance, the finite state approach fails. Previous work has shown that even in such setting decidability can be obtained by restricting the problem in various ways [1, 7].

- In XML document processing, regular concepts occur in various contexts. First, most applications restrict the structure of the allowed documents to conform to a certain specification (DTD or XML schema), 
which can be modeled as a regular tree language. Second, navigation (XPath) and transformation (XSLT) languages are tightly connected to various tree automata models and other regular description mechanism, see, for example, [12].

All these approaches concentrate on the structure of the XML documents and ignore the attribute and text values. From a database point of view, this is not completely satisfactory, because a schema should allow one not only to describe the structure of the data, but also to define restrictions on the data values via integrity constraints such as key or inclusion constraints. There exist a work addressing this problem [2], but like in the case of model checking, the methods rely heavily on a case-to-case analysis.

So, in the above settings, the finite state abstraction leads to interesting results, but does not address all problems arising in applications. In both cases, it would be already a big advance, if each position, in either a string or a tree, could carry a data value, in addition to its label.

This paper is part of a broader research program which aims at studying such extensions in a systematic way. As any kind of operations on the infinite domain quickly leads to undecidability of basic processing tasks (even a linear order on the domain is harmful), we concentrate on the setting, where data values can only be tested for equality. Furthermore, in this paper we only consider finite data strings, that is, finite strings, where each position carries a label from a finite alphabet and a data value from an infinite domain. Recently, there has been a significant amount of work in this direction, see [3, 4, 6, 9, 13, 15].

Roughly speaking, there are two approaches to studying data languages: logic and automata. Below is a brief survey on both approaches. For a more comprehensive survey, we refer the reader to [15]. The study of data languages, which can also be viewed as languages over infinite alphabets, starts with the introduction of finite-memory automata (FMA) in [9], which are also known as register automata (RA). The study of RA was continued and extended in [13], in which pebble automata (PA) were also introduced. Each of both models has its own advantages and disadvantages. Languages accepted by FMA are closed under standard language operations: intersection, union, concatenation, and Kleene star. In addition, from the computational point of view, FMA are a much easier model to handle. Their emptiness problem is decidable, whereas the same problem for PA is not. However, the 
PA languages possess a very nice logical property: closure under all boolean operations, whereas FMA languages are not closed under complementation.

Later in [4] first-order logic for data languages was considered, and, in particular, the so-called data automata was introduced. It was shown that data automata define the fragment of existential monadic second order logic for data languages in which the first order part is restricted to two variables only. An important feature of data automata is that their emptiness problem is decidable, even for the infinite words, but is at least as hard as reachability for Petri nets. The automata themselves always work nondeterministically and seemingly cannot be determinized, see [3]. It was also shown that the satisfiability problem for the three-variable first order logic is undecidable.

Another logical approach is via the so called linear temporal logic with $n$ register freeze quantifier over the labels $\Sigma$, denoted $\operatorname{LTL}_{n}^{\downarrow}(\Sigma, \mathrm{X}, \mathrm{U})$, see [6]. It was shown that one way alternating $n$ register automata accept all $\operatorname{LTL}_{n}^{\downarrow}(\Sigma, \mathrm{X}, \mathrm{U})$ languages and the emptiness problem for one way alternating one register automata is decidable. Hence, the satisfiability problem for $\operatorname{LTL}_{1}^{\downarrow}(\Sigma, \mathrm{X}, \mathrm{U})$ is decidable as well. Adding one more register or past time operators to $\operatorname{LTL}_{1}^{\downarrow}(\Sigma, \mathrm{X}, \mathrm{U})$ makes the satisfiability problem undecidable.

In this paper we continue the study of PA, which are finite state automata with a finite number of pebbles. The pebbles are placed on/lifted from the input word in the stack discipline - first in last out - and are intended to mark positions in the input word. One pebble can only mark one position and the most recently placed pebble serves as the head of the automaton. The automaton moves from one state to another depending on the current label and the equality tests among data values in the positions currently marked by the pebbles, as well as, the equality tests among the positions of the pebbles.

Furthermore, as defined in [13], there are two types of PA, according to the position of the new pebble placed. In the first type, the ordinary PA, also called strong PA, the new pebbles are placed at the beginning of the string. In the second type, called weak PA, the new pebbles are placed at the position of the most recent pebble. Obviously, two-way weak PA is just as expressive as two-way ordinary PA. However, it is known that one-way nondeterministic weak PA are weaker than one-way ordinary PA, see [13, Theorem 4.5.].

We show that the emptiness problem for one-way weak 2-pebble automata is decidable, while the same problem for one-way weak 3-pebble automata is undecidable. We also introduce the so-called top view weak PA. Roughly 
speaking, top view weak PA are one-way weak PA where the equality test is performed only between the data values seen by the two most recently placed pebbles. Top view weak PA are quite robust: alternating, nondeterministic and deterministic top view weak PA have the same recognition power. To the best of our knowledge, this is the first model of computation for data language with such robustness. It is also shown that top view weak PA can be simulated by one-way alternating one-register RA. Therefore, their emptiness problem is decidable. Another interesting feature is top view weak PA can simulate all $\operatorname{LTL}_{1}^{\downarrow}(\Sigma, \mathrm{X}, \mathrm{U})$ languages, and the number of pebbles needed to simulate such LTL sentences corresponds linearly to the so called free quantifier rank of the sentences, the depth of the nesting level of the freeze operators in the sentence.

This paper is organized as follows. In Section 2 we review the models of computations for data languages considered in this paper. Section 3 and Section 4 deals with the decidability and the complexity issues of weak PA, respectively. In Section [6] we introduce top view weak PA. We also introduce a simple extension to top view weak PA, called unbounded top view weak PA, in which the number of pebbles is unbounded in Section 7 Finally, we end our paper with a brief observation in Section 8. This paper is augmented with appendices that contain most of the omitted details.

\section{Models of computations}

In Subsections 2.1 and 2.2 we recall the definition of weak PA from [13, and review the strict hierarchy of weak PA languages established in [16]. In Subsection 2.3 we recall the temporal logical framework for data languages.

We will use the following notation. We always denote by $\Sigma$ a finite alphabet of labels and by $\mathfrak{D}$ an infinite set of data values. A $\Sigma$-data word $w=\left(\begin{array}{c}\sigma_{1} \\ a_{1}\end{array}\right)\left(\begin{array}{c}\sigma_{2} \\ a_{2}\end{array}\right) \cdots\left(\begin{array}{c}\sigma_{n} \\ a_{n}\end{array}\right)$ is a finite sequence over $\Sigma \times \mathfrak{D}$, where $\sigma_{i} \in \Sigma$ and $a_{i} \in \mathfrak{D}$. A $\Sigma$-data language is a set of $\Sigma$-data words. The idea is that the alphabet $\Sigma$ is accessed directly, while data values can only be tested for equality.

We assume that neither of $\Sigma$ and $\mathfrak{D}$ contain the left-end marker $\triangleleft$ or the right-end marker $\triangleright$. The input word to the automaton is of the form $\triangleleft w \triangleright$, where $\triangleleft$ and $\triangleright$ mark the left-end and the right-end of the input word.

We will also use the following notations. For $w=\left(\begin{array}{c}\sigma_{1} \\ a_{1}\end{array}\right) \cdots\left(\begin{array}{c}\sigma_{n} \\ a_{n}\end{array}\right)$,

$$
\operatorname{Proj}_{\Sigma}(w)=\sigma_{1} \cdots \sigma_{n}
$$




$$
\begin{aligned}
\operatorname{Proj}_{\mathfrak{D}}(w) & =a_{1} \cdots a_{n} \\
\operatorname{Cont}_{\Sigma}(w) & =\left\{\sigma_{1}, \ldots, \sigma_{n}\right\} \\
\operatorname{Cont}_{\mathfrak{D}}(w) & =\left\{a_{1}, \ldots, a_{n}\right\}
\end{aligned}
$$

Finally, the symbols $\nu, \vartheta, \sigma, \ldots$, possibly indexed, denote labels in $\Sigma$ and the symbols $a, b, c, d, \ldots$, possibly indexed, denote data values in $\mathfrak{D}$.

\subsection{Pebble automata}

Definition 1 (See [13, Definition 2.3]) A one-way alternating weak k-pebble automaton or, in short, $k$-PA, over $\Sigma$ is a system $\mathcal{A}=\left\langle\Sigma, Q, q_{0}, F, \mu, U\right\rangle$ whose components are defined as follows.

- $Q, q_{0} \in Q$ and $F \subseteq Q$ are a finite set of states, the initial state, and the set of final states, respectively;

- $U \subseteq Q-F$ is the set of universal states; and

- $\mu \subseteq \mathcal{C} \times \mathcal{D}$ is the transition relation, where

$-\mathcal{C}$ is a set whose elements are of the form $(i, \sigma, V, q)$ where $1 \leq i \leq$ $k, \sigma \in \Sigma, V \subseteq\{i+1, \ldots, k\}$ and $q \in Q ;$ and

- $\mathcal{D}$ is a set whose elements are of the form ( $q$, act), where $q \in Q$ and act $\in\{$ stay, right, place-pebble, lift-pebble $\}$.

Elements of $\mu$ will be written as $(i, \sigma, V, q) \rightarrow(p$, act $)$.

Remark 2 Note that the pebble numbering that differs from that in [13]. In the above definition we adopt the pebble numbering from [5] in which the pebbles placed on the input word are numbered from $k$ to $i$ and not from 1 to $i$ as in [13]. The reason for this reverse numbering is that it allows us to view the computation between placing and lifting pebble $i$ as a computation of an $(i-1)$-pebble automaton.

Furthermore, the automaton is no longer equipped with the ability to compare positional equality, in contrast with the ordinary PA introduced in [13]. Such ability no longer makes any difference because the new pebbles are placed in the "weak" manner. 
Given a word $w=\left(\begin{array}{c}\sigma_{1} \\ a_{1}\end{array}\right) \cdots\left(\begin{array}{c}\sigma_{n} \\ a_{n}\end{array}\right) \in(\Sigma \times \mathfrak{D})^{*}$, a configuration of $\mathcal{A}$ on $\triangleleft w \triangleright$ is a triple $[i, q, \theta]$, where $i \in\{1, \ldots, k\}, q \in Q$, and $\theta:\{i, i+1, \ldots, k\} \rightarrow$ $\{0,1, \ldots, n, n+1\}$, where 0 and $n+1$ are positions of the end markers $\triangleleft$ and $\triangleright$, respectively. The function $\theta$ defines the position of the pebbles and is called the pebble assignment. The initial configuration is $\gamma_{0}=\left[k, q_{0}, \theta_{0}\right]$, where $\theta_{0}(k)=0$ is the initial pebble assignment. A configuration $[i, q, \theta]$ with $q \in F$ is called an accepting configuration.

A transition $(i, \sigma, V, p) \rightarrow \beta$ applies to a configuration $[j, q, \theta]$, if

(1) $i=j$ and $p=q$,

(2) $V=\left\{l>i: a_{\theta(l)}=a_{\theta(i)}\right\}$, and

(3) $\sigma_{\theta(i)}=\sigma$.

Next we define the transition relation $\vdash_{\mathcal{A}}$ as follows: $[i, q, \theta] \vdash_{\mathcal{A}}\left[i^{\prime}, q^{\prime}, \theta^{\prime}\right]$, if there is a transition $\alpha \rightarrow(p$, act $) \in \mu$ that applies to $[i, q, \theta]$ such that $q^{\prime}=p$, for all $j>i, \theta^{\prime}(j)=\theta(j)$, and

- if act = stay, then $i^{\prime}=i$ and $\theta^{\prime}(i)=\theta(i)$,

- if act $=$ right, then $i^{\prime}=i$ and $\theta^{\prime}(i)=\theta(i)+1$,

- if act $=$ lift-pebble, then $i^{\prime}=i+1$,

- if act $=$ place-pebble, then $i^{\prime}=i-1, \theta^{\prime}(i-1)=\theta(i)$ and $\theta^{\prime}(i)=\theta(i)$.

As usual, we denote the reflexive transitive closure of $\vdash_{\mathcal{A}}$ by $\vdash_{\mathcal{A}}^{*}$. When the automaton $\mathcal{A}$ is clear from the context, we shall omit the subscript $\mathcal{A}$.

The acceptance criteria is based on the notion of leads to acceptance below. For every configuration $\gamma=[i, q, \theta]$,

- if $q \in F$, then $\gamma$ leads to acceptance;

- if $q \in U$, then $\gamma$ leads to acceptance if and only if for all configurations $\gamma^{\prime}$ such that $\gamma \vdash \gamma^{\prime}, \gamma^{\prime}$ leads to acceptance;

- if $q \notin F \cup U$, then $\gamma$ leads to acceptance if and only if there is at least one configuration $\gamma^{\prime}$ such that $\gamma \vdash \gamma^{\prime}$, and $\gamma^{\prime}$ leads to acceptance. 
A $\Sigma$-data word $w \in(\Sigma \times \mathfrak{D})^{*}$ is accepted by $\mathcal{A}$, if $\gamma_{0}$ leads to acceptance. The language $L(\mathcal{A})$ consists of all data words accepted by $\mathcal{A}$.

The automaton $\mathcal{A}$ is nondeterministic, if the set $U=\emptyset$, and it is deterministic, if there is exactly one transition that applies for each configuration. It turns out that weak PA languages are quite robust.

Theorem 3 For all $k \geq 1$, alternating, non-deterministic and deterministic weak $k$-PA have the same recognition power.

The proof is quite standard. For the details of the proof, we refer the reader to Appendix D.

Next, we define the hierarchy of languages accepted by PA. For $k \geq 1$, We define the following classes of languages.

$$
\begin{aligned}
\mathrm{wPA}_{k} & =\{L: L \text { is accepted by a weak } k-\mathrm{PA}\} ; \text { and } \\
\mathrm{wPA} & =\bigcup_{k \geq 1} \mathrm{wPA}_{k}
\end{aligned}
$$

This example will be useful in the subsequent section.

Example 4 Consider a $\Sigma$-data language $L_{\sim}$ defined as follows. A $\Sigma$-data word $w=\left(\begin{array}{c}\sigma_{1} \\ a_{1}\end{array}\right) \cdots\left(\begin{array}{c}\sigma_{n} \\ a_{n}\end{array}\right) \in L_{\sim}$ if and only if for all $i, j=1, \ldots, n$, if $a_{i}=a_{j}$, then $\sigma_{i}=\sigma_{j}$. That is, $w \in L_{\sim}$ if and only if whenever two positions in $w$ carry the same data value, their labels are the same.

The language $L_{\sim}$ is accepted by weak 2-PA which works in the following manner. Pebbles 2 iterates through all possible positions in $w$. At each iteration, pebble 1 is placed and scans through all the positions to the right of pebble 2, checking whether there is a position with the same data value of pebble 2. If there is such position, then the labels seen by pebbles 1 and 2 are the same.

\subsection{Strict hierarchy of weak PA languages}

In this section we review an example of data language introduced in [16]. It will be useful in establishing our definability results for $\operatorname{LTL}_{1}^{\downarrow}(\Sigma, \mathrm{X}, \mathrm{U})$ languages. 
Let $\Sigma=\{\sigma\}$ be a singleton alphabet. For an integer $m \geq 1$, the language $\mathcal{R}_{m}^{+}$consists of $\Sigma$-data words of the form

$$
\left(\begin{array}{c}
\sigma \\
a_{0}
\end{array}\right)\left(\begin{array}{c}
\sigma \\
a_{1}
\end{array}\right) \underbrace{\ldots}_{w_{1}}\left(\begin{array}{c}
\sigma \\
a_{1}
\end{array}\right)\left(\begin{array}{c}
\sigma \\
a_{2}
\end{array}\right) \ldots \ldots \ldots\left(\begin{array}{c}
\sigma \\
a_{m-2}
\end{array}\right)\left(\begin{array}{c}
\sigma \\
a_{m-1}
\end{array}\right) \underbrace{\ldots}_{w_{m-1}}\left(\begin{array}{c}
\sigma \\
a_{m-1}
\end{array}\right)\left(\begin{array}{c}
\sigma \\
a_{m}
\end{array}\right)
$$

where

- for each $i=0,1, \ldots, m-1, a_{i} \neq a_{i+1}$;

- for each $i=1, \ldots, m-1, a_{i} \notin \operatorname{Cont}_{\mathfrak{D}}\left(w_{i}\right)$.

The language $\mathcal{R}^{+}$is defined as

$$
\mathcal{R}^{+}=\bigcup_{m=1,2, \ldots} \mathcal{R}_{m}^{+}
$$

Theorem 5 (See [16, Lemma 18].) For each $k=1,2, \ldots$,

1. $\mathcal{R}_{k} \in w P A_{k}$ and $\mathcal{R}_{k+1} \notin w P A_{k}$;

2. $w P A_{k} \subsetneq w P A_{k+1}$.

\subsection{Linear temporal logic with one register freeze quan- tifier}

In this section we recall the definition of Linear Temporal Logic (LTL) with one register freeze quantifier [6]. We consider only one-way temporal operators "next" $\mathrm{X}$ and "until" $\mathrm{U}$, and do not consider their past time counterparts.

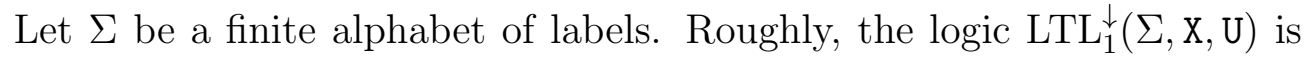
standard LTL augmented with a register to store a data value. Formally, the formulas are defined as follows.

- Both True and False belong to $\operatorname{LTL}_{1}^{\downarrow}(\Sigma, \mathrm{X}, \mathrm{U})$.

- The empty formula $\epsilon$ belongs to $\operatorname{LTL}_{1}^{\downarrow}(\Sigma, \mathrm{X}, \mathrm{U})$.

- For each $\sigma \in \Sigma, \sigma$ is in $\operatorname{LTL}_{1}^{\downarrow}(\Sigma, \mathrm{X}, \mathrm{U})$.

- If $\varphi, \psi$ are in $\operatorname{LTL}_{1}^{\downarrow}(\Sigma, \mathrm{X}, \mathrm{U})$, then so are $\neg \varphi, \varphi \vee \psi$ and $\varphi \wedge \psi$.

- $\uparrow$ is in $\operatorname{LTL}_{1}^{\downarrow}(\Sigma, \mathrm{X}, \mathrm{U})$. 
- If $\varphi$ is in $\operatorname{LTL}_{1}^{\downarrow}(\Sigma, \mathrm{X}, \mathrm{U})$, then so is $\mathrm{X} \varphi$.

- If $\varphi$ is in $\operatorname{LTL}_{1}^{\downarrow}(\Sigma, \mathrm{X}, \mathrm{U})$, then so is $\downarrow \varphi$.

- If $\varphi, \psi$ are in $\operatorname{LTL}_{1}^{\downarrow}(\Sigma, \mathrm{X}, \mathrm{U})$, then so is $\varphi \mathrm{U} \psi$.

Intuitively, the predicate $\uparrow$ is intended to mean that the current data value is the same as the data value in the register, while $\downarrow \varphi$ is intended to mean that the formula $\varphi$ holds when the register contains the current data value. This will be made precise in the definition of the semantics of $\operatorname{LTL}_{1}^{\downarrow}(\Sigma, \mathrm{X}, \mathrm{U})$ below.

An occurrence of $\uparrow$ within the scope of some freeze quantification $\downarrow$ is bounded by it; otherwise, it is free. A sentence is a formula with no free occurrence of $\uparrow$.

Next we define the freeze quantifier rank of a sentence $\varphi$, denoted by $\operatorname{fqr}(\varphi)$.

- For each $\sigma \in \Sigma, \operatorname{fqr}(\sigma)=0$.

- $\operatorname{fqr}(\operatorname{True})=\mathrm{fqr}($ False $)=\mathrm{fqr}(\uparrow)=0$.

- $\operatorname{fgr}(\mathrm{X} \varphi)=\operatorname{fgr}(\neg \varphi)=\operatorname{fgr}(\varphi)$, for every $\varphi$ in $\operatorname{LTL}_{1}^{\downarrow}(\Sigma, \mathrm{X}, \mathrm{U})$.

- $\operatorname{fgr}(\varphi \vee \psi)=\operatorname{fgr}(\varphi \wedge \psi)=\operatorname{fqr}(\varphi \mathrm{U} \psi)=\max (\operatorname{fgr}(\varphi), \operatorname{fgr}(\psi))$, for every $\varphi$ and $\psi$ in $\operatorname{LTL}_{1}^{\downarrow}(\Sigma, \mathrm{X}, \mathrm{U})$.

- $\operatorname{fgr}(\downarrow \varphi)=\operatorname{far}(\varphi)+1$, for every $\varphi$ in $\operatorname{LTL}_{1}^{\downarrow}(\Sigma, \mathrm{X}, \mathrm{U})$.

Finally, we define the semantics of $\operatorname{LTL}_{1}^{\downarrow}(\Sigma, \mathrm{X}, \mathrm{U})$. Let $w=\left(\begin{array}{c}\sigma_{1} \\ a_{1}\end{array}\right) \cdots\left(\begin{array}{c}\sigma_{n} \\ a_{n}\end{array}\right)$ be a $\Sigma$-data word. For a position $i=1, \ldots, n$, a data value $a$ and a formula $\varphi$ in $\operatorname{LTL}_{1}^{\downarrow}(\Sigma, \mathrm{X}, \mathrm{U}), w, i \models_{a} \varphi$ means that $\varphi$ is satisfied by $w$ at position $i$ when the content of the register is $a$. As usual, $w, i \nvdash_{a} \varphi$ means $\varphi$ is not satisfied by $w$ at position $i$ when the content of the register is $a$. The satisfaction relation is defined inductively as follows.

- $w, i \models_{a} \epsilon$ for all $i=1,2, \ldots, n$ and $a \in \mathfrak{D}$.

- $w, i \models_{a}$ True and $w, i \not \nvdash_{a}$ False, for all $i=1,2,3, \ldots$ and $a \in \mathfrak{D}$.

- $w, i \models_{a} \sigma$ if and only if $\sigma_{i}=\sigma$.

- $w, i \models_{a} \varphi \vee \psi$ if and only if $w, i \models_{a} \varphi$ or $w, i \models_{a} \psi$. 
- $w, i \models_{a} \varphi \wedge \psi$ if and only if $w, i \models_{a} \varphi$ and $w, i \models_{a} \psi$.

- $w, i \models_{a} \neg \varphi$ if and only if $w, i \nvdash_{a} \varphi$.

- $w, i \models_{a} \mathbf{X} \varphi$ if and only if $1 \leq i<|w|$ and $w, i+1 \models_{a} \varphi$.

- $w, i \models_{a} \varphi \mathrm{U} \psi$ if and only if there exists $j \geq i$ such that

$-w, j \models_{a} \psi$ and

$-w, j^{\prime} \models_{a} \varphi$, for all $j^{\prime}=i, \ldots, j-1$.

- $w, i \models_{a} \downarrow \varphi$ if and only if $w, i \models_{a_{i}} \varphi$

- $w, i \models_{a} \uparrow$ if and only if $a=a_{i}$.

For a sentence $\varphi$ in $\operatorname{LTL}_{1}^{\downarrow}(\Sigma, \mathrm{X}, \mathrm{U})$, we define the $\Sigma$-data language $L(\varphi)$ by

$$
L(\varphi)=\left\{w \mid w, 1 \models_{a} \varphi \text { for some } a \in \mathfrak{D}\right\} .
$$

Note that since $\varphi$ is a sentence, all occurrences of $\uparrow$ in $\varphi$ are bounded. Thus, it makes no difference which data value $a$ is used in the statement $w, 1 \models_{a} \varphi$ of the definition of $L(\varphi)$.

\section{$3 \quad$ Decidability and undecidability of weak PA}

In this section we will discuss the decidability issue of weak PA. We show that the emptiness problem for weak 3-PA is undecidable, while the same problem for weak 2-PA is decidable. The proof of the decidability of the emptiness problem for weak 2-PA will be the basis of the proof of the decidability of the same problem for top view weak PA.

Theorem 6 The emptiness problem for weak 3-PA is undecidable.

Proof. The proof is very similar to the proof of the undecidability of the emptiness problem for weak 5-PA in [13]. We observe that the same proof can be easily adopted to weak 3-PA. The details are provided below. It uses a reduction from the Post Correspondence Problem (PCP), which is well known to be undecidable [8]. An instance of PCP is a sequence of pairs $\left(x_{1}, y_{1}\right), \ldots,\left(x_{n}, y_{n}\right)$, where each $x_{1}, y_{1}, \ldots, x_{n}, y_{n} \in\{\alpha, \beta\}^{*}$. 
This instance has a solution if there exist indexes $i_{1}, \ldots, i_{m} \in\{1, \ldots, n\}$ such that $x_{i_{1}} \cdots x_{i_{m}}=y_{i_{1}} \cdots y_{i_{m}}$. The PCP asks whether a given instance of the problem has a solution.

In the following we show how to encode a solution of an instance of PCP into a data word which possesses properties that can be checked by a weak 3-PA. Let $\Sigma=\{1, \ldots, n, \alpha, \beta, \$\}$. We denote by $x_{i}=\nu_{i, 1} \cdots \nu_{i, l_{i}, l_{i}}$, for each $i=1, \ldots, n$. Each string $x_{i}$ is encoded as $\operatorname{Enc}\left(x_{i}\right)=\left(\begin{array}{l}\nu_{i, 1} \\ a_{i, 1}\end{array}\right) \cdots\left(\begin{array}{l}\nu_{i, l_{i}} \\ a_{i, l_{i}}\end{array}\right)$ where $a_{i, 1}, \ldots, a_{i, l_{i}}$ are pairwise different.

The string $x_{i_{1}} x_{i_{2}} \cdots x_{i_{m}}$ can be encoded as

$$
\operatorname{Enc}\left(x_{i_{1}}, x_{i_{2}}, \ldots, x_{i_{m}}\right)=\left(\begin{array}{c}
i_{1} \\
b_{1}
\end{array}\right) \operatorname{Enc}\left(x_{i_{1}}\right)\left(\begin{array}{c}
i_{2} \\
b_{2}
\end{array}\right) \operatorname{Enc}\left(x_{i_{2}}\right) \cdots\left(\begin{array}{l}
i_{m} \\
b_{m}
\end{array}\right) \operatorname{Enc}\left(x_{i_{m}}\right)
$$

where all the data values that appear in it are pairwise different. Note that even if $i_{j}=i_{j^{\prime}}$ for some $j, j^{\prime}$, the data values that appear in $\operatorname{Enc}\left(x_{i_{j}}\right)$ do not appear in $\operatorname{Enc}\left(x_{i_{j^{\prime}}}\right)$ and vice versa. The idea is each data value is used to mark a place in the string.

Similarly, the string $y_{j_{1}} y_{j_{2}} \cdots y_{j_{l}}$ can be encoded as

$$
\operatorname{Enc}\left(y_{j_{1}}, y_{j_{2}}, \ldots, y_{j_{l}}\right)=\left(\begin{array}{l}
j_{1} \\
c_{1}
\end{array}\right) \operatorname{Enc}\left(y_{j_{1}}\right)\left(\begin{array}{l}
j_{2} \\
c_{2}
\end{array}\right) \operatorname{Enc}\left(y_{j_{2}}\right) \cdots\left(\begin{array}{l}
j_{l} \\
c_{l}
\end{array}\right) \operatorname{Enc}\left(y_{j_{l}}\right)
$$

where the data values that appear in it are pairwise different.

Now the data word

$$
\left(\begin{array}{l}
i_{1} \\
b_{1}
\end{array}\right) \operatorname{Enc}\left(x_{i_{1}}\right) \cdots\left(\begin{array}{l}
i_{m} \\
b_{m}
\end{array}\right) \operatorname{Enc}\left(x_{i_{m}}\right)\left(\begin{array}{l}
\$ \\
d
\end{array}\right)\left(\begin{array}{l}
j_{1} \\
c_{1}
\end{array}\right) \operatorname{Enc}\left(y_{j_{1}}\right) \cdots\left(\begin{array}{l}
j_{l} \\
c_{l}
\end{array}\right) \operatorname{Enc}\left(y_{j_{l}}\right)
$$

constitutes a solution to the instance of PCP if and only if

$$
\begin{aligned}
i_{1} i_{2} \cdots i_{m} & =j_{1} j_{2} \cdots j_{l} \\
\operatorname{Proj}_{\Sigma}\left(\operatorname{Enc}\left(x_{i_{1}}\right) \cdots \operatorname{Enc}\left(x_{i_{m}}\right)\right) & =\operatorname{Proj}_{\Sigma}\left(\operatorname{Enc}\left(y_{j_{1}}\right) \cdots \operatorname{Enc}\left(y_{j_{l}}\right)\right)
\end{aligned}
$$

Now, in order to able to check such property with weak 3-PA, we demand the following additional criteria.

1. $b_{1} \cdots b_{m}=c_{1} \cdots c_{l}$;

2. $\operatorname{Proj}_{\mathfrak{D}}\left(\operatorname{Enc}\left(x_{i_{1}}\right) \cdots \operatorname{Enc}\left(x_{i_{m}}\right)\right)=\operatorname{Proj}_{\mathfrak{D}}\left(\operatorname{Enc}\left(y_{j_{1}}\right) \cdots \operatorname{Enc}\left(y_{j_{l}}\right)\right)$ 
3. For any two positions $h_{1}$ and $h_{2}$ where $h_{1}$ is to the left of the delimiter $\left(\begin{array}{l}\$ \\ c\end{array}\right)$ and $h_{2}$ is to the right of the delimiter $\left(\begin{array}{l}\$ \\ c\end{array}\right)$, if both of them have the same data value, then both of them are labelled with the same label.

All the Criterias (1)-(3) imply Equations 1 and 2.

Because the data values that appears in $\operatorname{Proj}_{\mathfrak{D}}\left(\operatorname{Enc}\left(x_{i_{1}}\right), \ldots, \operatorname{Enc}\left(x_{i_{m}}\right)\right)$ are pairwise different, all of them are checkable by three pebbles in the "weak" manner. For example, to check Criteria (1), the automaton does the following.

- Check that $b_{1}=c_{1}$.

- Check that for each $i=1, \ldots, m-1$, there exists $j$ such that $a_{i} a_{i+1}=$ $b_{j} b_{j+1}$.

It can be done by placing pebble 3 to read $a_{i}$ and pebble 2 to read $a_{i+1}$, then using pebble 3 to search on the other side of $\$$ for the index $j$.

- Finally, check that $b_{m}=c_{l}$.

Criteria (2) can be checked similarly and Criteria (3) is straightforward. The reduction is now complete and we prove that the emptiness problem for weak 3-PA is undecidable.

Now we are going to show that the emptiness problem for weak 2-PA is decidable. The proof is by simulating weak 2-PA by one-way alternating one register automata (1-RA). In fact, the simulation can be easily generalized to arbitrary number of pebbles. That is, weak $k$-PA can be simulated by one-way alternating $(k-1)$-RA. This result settles a question left open in [13]: Can weak PA be simulated by alternating RA? We refer the reader to Appendix $\mathrm{C}$ for the details of the proof.

Theorem 7 For every weak 2-PA $\mathcal{A}$, there exists a one-way alternating 1$R A \mathcal{A}^{\prime}$ such that $L(\mathcal{A})=L\left(\mathcal{A}^{\prime}\right)$. Moreover, the construction of $\mathcal{A}^{\prime}$ from $\mathcal{A}$ is effective.

Now, by Theorem [7, we immediately obtain the decidability of weak 2-PA because the emptiness problem for one-way alternating 1-RA is decidable [6, Theorem 4.4].

Corollary 8 The emptiness problem for weak 2-PA is decidable. 
We devote the rest of this section to the proof of Theorem 7 .

Let $\mathcal{A}=\left\langle Q, q_{0}, \mu, F\right\rangle$ be a weak 2-PA. We assume that $\mathcal{A}$ is deterministic. Furthermore, we normalize the behavior of $\mathcal{A}$ as follows.

- Pebble 1 is lifted only after it reads the right-end marker symbol $\triangleright$.

- Only pebble 2 can enter a final state and it does so after it reads the right-end marker $\triangleright$.

- Immediately after pebble 2 moves right, pebble 1 is placed.

- Immediately after pebble 1 is lifted, pebble 2 moves right.

On input word $w=\left(\begin{array}{c}\sigma_{1} \\ d_{1}\end{array}\right) \cdots\left(\begin{array}{l}\sigma_{n} \\ d_{n}\end{array}\right)$, the run of $\mathcal{A}$ on $\triangleleft w \triangleright$ can be depicted as a tree shown in Figure 3 .

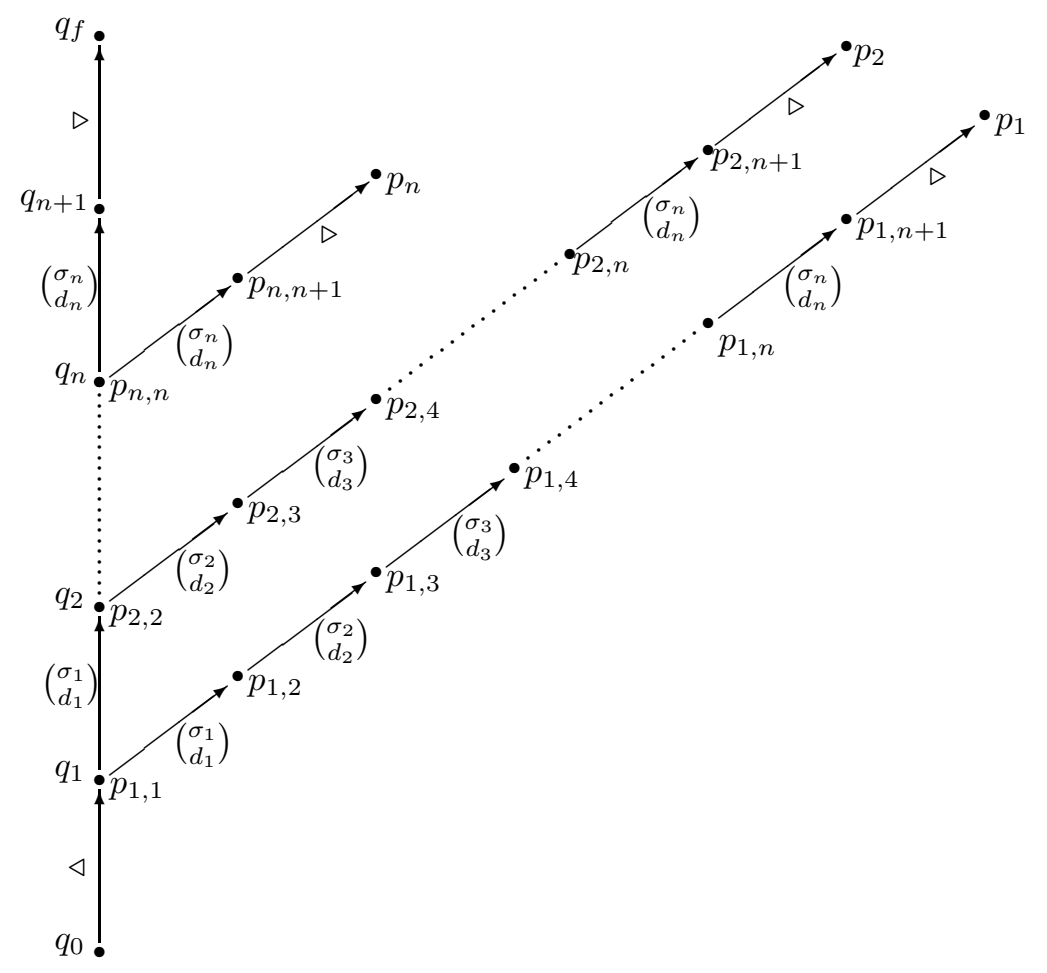

Figure 1: The tree representation of a run of $\mathcal{A}$ on $w=\left(\begin{array}{c}\sigma_{1} \\ d_{1}\end{array}\right) \cdots\left(\begin{array}{l}\sigma_{n} \\ d_{n}\end{array}\right)$.

The meaning of the tree is as follows. 
- $q_{0}, q_{1}, \ldots, q_{n}, q_{n+1}$ are the states of $\mathcal{A}$ when pebble 2 is the head pebble reading the positions $0,1, \ldots, n, n+1$, respectively, that is, the symbols $\triangleleft,\left(\begin{array}{c}\sigma_{1} \\ d_{1}\end{array}\right), \ldots,\left(\begin{array}{c}\sigma_{n} \\ d_{n}\end{array}\right), \triangleright$, respectively.

- $q_{f}$ is the state of $\mathcal{A}$ after pebble 2 reads the symbol $\triangleright$.

- For $1 \leq i \leq j \leq n, p_{i, j}$ is the state of $\mathcal{A}$ when pebble 1 is the head pebble above the position $j$ while pebble 2 is above the position $i$.

- For $1 \leq i \leq n$, the state $p_{i}$ is the state of $\mathcal{A}$ immediately after pebble 1 is lifted and pebble 2 is above the position $i$.

It must be noted that there is a transition $\left(2, \sigma_{i}, \emptyset, p_{i}\right) \rightarrow\left(q_{i+1}\right.$, right $)$ applied by $\mathcal{A}$ that is not depicted in the figure.

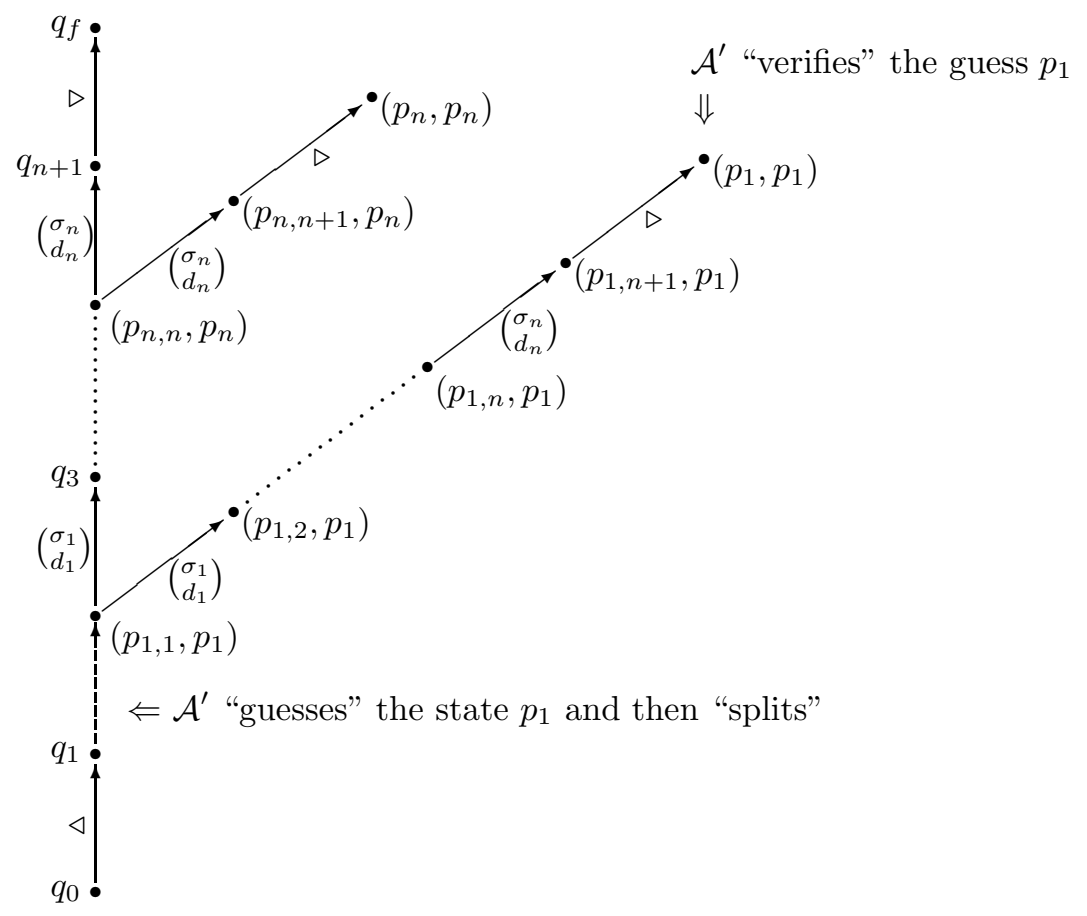

Figure 2: The corresponding run of $\mathcal{A}^{\prime}$ to the one in Figure 3 , 
Now the simulation of $\mathcal{A}$ by a one-way alternating 1-RA $\mathcal{A}^{\prime}$ becomes straightforward by transforming the tree in Figure 3 into a tree depicting the computation of $\mathcal{A}^{\prime}$ on the same word $w$.

Roughly, the automaton $\mathcal{A}^{\prime}$ is defined as follows.

- The states of $\mathcal{A}^{\prime}$ are elements of $Q \cup(Q \times Q) 1$;

- the initial state is $q_{0}$; and

- the set of final states is $F \cup\{(p, p): p \in Q\}$.

For each placement of pebble 1 on position $i$, the automaton performs the following "Guess-Split-Verify" procedure which consists of the following steps.

1. From the state $q_{i}, \mathcal{A}^{\prime}$ "guesses" the state in which pebble 1 is eventually lifted, i.e. the state $p_{i}$, and stores it in its internal state.

That is, $\mathcal{A}^{\prime}$ enters into the state $\left(q_{i}, p_{i}\right)$.

2. $\mathcal{A}^{\prime}$ "splits" its computation (conjunctively) into two branches.

- In one branch, assuming that the guess $p_{i}$ is correct, $\mathcal{A}^{\prime}$ moves right and enters into the state $q_{i+1}$, simulating the transition $\left(2, \emptyset, p_{i}\right) \rightarrow$ $\left(q_{i+1}\right.$, right $)$. After this, it recursively performs the Guess-SplitVerify procedure for the next placement of pebble 1 on position $(i+$ 1).

- In the other branch $\mathcal{A}^{\prime}$ stores the data value $d_{i}$ in its register and simulates the run of pebble 1 on $\left(\begin{array}{l}\sigma_{i} \\ d_{i}\end{array}\right) \cdots\left(\begin{array}{l}\sigma_{n} \\ d_{n}\end{array}\right)$ to "verify" that the guess $p_{i}$ is correct.

That is, $\mathcal{A}^{\prime}$ accepts only if it ends in the state $\left(p_{i}, p_{i}\right)$.

Figure 3 shows the corresponding run of $\mathcal{A}^{\prime}$ on the same word.

\section{Complexity of weak 2-PA}

In this subsection we are going to determine the time complexity of three specific problems related to weak 2-PA.

\footnotetext{
${ }^{1}$ Actually $\mathcal{A}^{\prime}$ needs some other auxiliary states. However, for the intuitive explanation here the set $Q \cup(Q \times Q)$ suffices. We refer the reader to Appendix $\mathbb{C}$ for the details.
} 
Emptiness problem. The emptiness problem for weak 2-PA. That is, given a weak 2 -PA $\mathcal{A}$, is $L(\mathcal{A})=\emptyset$ ?

Labelling problem. Given a weak 2-PA $\mathcal{A}$ over the labels $\Sigma$ and a sequence of data values $d_{1} \cdots d_{n} \in \mathfrak{D}^{n}$, is there a sequence of labels $\sigma_{1} \cdots \sigma_{n} \in \Sigma^{n}$ such that $\left(\begin{array}{c}\sigma_{1} \\ d_{1}\end{array}\right) \cdots\left(\begin{array}{c}\sigma_{n} \\ d_{n}\end{array}\right) \in L(\mathcal{A})$ ?

Data value membership problem. Given a weak 2-PA $\mathcal{A}$ over the labels $\Sigma$ and a sequence of finite labels $\sigma_{1} \cdots \sigma_{n} \in \Sigma^{n}$, is there a sequence of data values $d_{1} \cdots d_{n} \in \mathfrak{D}^{n}$ such that $\left(\begin{array}{c}\sigma_{1} \\ d_{1}\end{array}\right) \cdots\left(\begin{array}{c}\sigma_{n} \\ d_{n}\end{array}\right) \in L(\mathcal{A})$ ?

The emptiness problem, as we have seen in the previous section, is decidable. The labelling and data value membership problem are definitely decidable. To solve the labelling problem, one simply iterates all possible sequence $\sigma_{1} \cdots \sigma_{n} \in \Sigma^{n}$ and runs $\mathcal{A}$ to check whether $\left(\begin{array}{c}\sigma_{1} \\ d_{1}\end{array}\right) \cdots\left(\begin{array}{c}\sigma_{n} \\ d_{n}\end{array}\right) \in L(\mathcal{A})$. Such straightforward algorithm requires $O\left(|\Sigma|^{n} \cdot n^{2}\right)$ computational steps. Similarly, to solve the data value membership problem, one can iterate all possible sequence of data values $d_{1} \cdots d_{n}$ and run $\mathcal{A}$ to check whether $\left(\begin{array}{c}\sigma_{1} \\ d_{1}\end{array}\right) \cdots\left(\begin{array}{l}\sigma_{n} \\ d_{n}\end{array}\right) \in L(\mathcal{A})$. Since the word is of length $n$, one simply needs to consider up to $n$ different data values. Such algorithm takes $O\left(n^{n} \cdot n^{2}\right)$ computational steps.

We are going to show that the emptiness problem is not primitive recursive, while both the labelling and data value membership problems are NP-complete.

We start the proof with a few simple examples of languages accepted by weak 2-PA. Though simple, they are very crucial in determining the complexity of the emptiness problem for weak 2-PA.

Example 9 Let $\Sigma=\{\alpha, \beta\}$. We define the $\Sigma$-data language $L_{\text {inc }}$ which consists of the data words of the following form:

$$
\underbrace{\left(\begin{array}{c}
\alpha \\
a_{1}
\end{array}\right) \cdots\left(\begin{array}{c}
\alpha \\
a_{m}
\end{array}\right)}_{w_{1}} \underbrace{\left(\begin{array}{c}
\beta \\
b_{1}
\end{array}\right) \cdots\left(\begin{array}{c}
\beta \\
b_{n}
\end{array}\right)}_{w_{2}},
$$

where

- the data values $a_{1}, \ldots, a_{m}$ are pairwise different;

- the data values $b_{1}, \ldots, b_{n}$ are pairwise different; 
- $\operatorname{Proj}_{\Sigma}\left(w_{1}\right)=\alpha^{m}$

- $\operatorname{Proj}_{\Sigma}\left(w_{2}\right)=\beta^{n}$

- $\operatorname{Cont}_{\mathfrak{D}}\left(w_{1}\right) \subseteq \operatorname{Cont}_{\mathfrak{D}}\left(w_{2}\right)$.

All these conditions can be checked by weak 2-PA. The intention of data words in $L_{i n c}$ is to represent the inequality $m \leq n$.

Example 10 Let $\Sigma=\{\alpha, \beta\}$. For a fixed $l \geq 0$, we define the language $L_{i n c,+1}$ which consists of the data words of the following form:

$$
\underbrace{\left(\begin{array}{c}
\alpha \\
a_{1}
\end{array}\right) \cdots\left(\begin{array}{c}
\alpha \\
a_{m}
\end{array}\right)}_{w_{1}} \underbrace{\left(\begin{array}{l}
\beta \\
b_{1}
\end{array}\right) \cdots\left(\begin{array}{c}
\beta \\
b_{n}
\end{array}\right)}_{w_{2}}
$$

where

- the data values $a_{1}, \ldots, a_{m}$ are pairwise different;

- the data values $b_{1}, \ldots, b_{n}$ are pairwise different;

- $\operatorname{Proj}_{\Sigma}\left(w_{1}\right)=\alpha^{m}$;

- $\operatorname{Proj}_{\Sigma}\left(w_{2}\right)=\beta^{n}$

- For each $a_{i} \in \operatorname{Cont}_{\mathfrak{D}}\left(w_{1}\right), a_{i} \neq b_{1}$.

- $\left\{a_{1}, \ldots, a_{m}\right\} \subseteq\left\{b_{2}, \ldots, b_{n}\right\}$.

Again, all these conditions can be checked by weak 2-PA. The intention of data words in $L_{i n c,+1}$ is to represent the inequality $m+1 \leq n$.

Example 11 Let $\Sigma=\{\alpha, \beta\}$. For a fixed $l \geq 0$, we define the language $L_{i n c,-1}$ which consists of the data words of the following form:

$$
\underbrace{\left(\begin{array}{c}
\alpha \\
a_{1}
\end{array}\right) \cdots\left(\begin{array}{c}
\alpha \\
a_{m}
\end{array}\right)}_{w_{1}} \underbrace{\left(\begin{array}{l}
\beta \\
b_{1}
\end{array}\right) \cdots\left(\begin{array}{c}
\beta \\
b_{n}
\end{array}\right)}_{w_{2}}
$$

where 
- the data values $a_{1}, \ldots, a_{m}$ are pairwise different;

- the data values $b_{1}, \ldots, b_{n}$ are pairwise different;

- $\operatorname{Proj}_{\Sigma}\left(w_{1}\right)=\alpha^{m}$;

- $\operatorname{Proj}_{\Sigma}\left(w_{2}\right)=\beta^{n}$;

- The symbol $a_{1} \notin\left\{b_{1}, \ldots, b_{n}\right\}$;

- For each $i=2, \ldots, m, a_{i} \in\left\{b_{1}, \ldots, b_{n}\right\}$.

Again, all these conditions can be checked by weak 2-PA. The intention of data words in $L_{i n c,-1}$ is to represent the inequality $m-1 \leq n$.

Theorem 12 The emptiness problem for weak 2-PA is not primitive recursive.

Proof. The proof is by simulation of incrementing counter automata. It follows closely the proof of similar lower bound for one-way alternating 1RA [6, Theorem 2.9]. It is known that the emptiness problem for incrementing counter automata is decidable [11, Theorem 6], but not primitive recursive [14. We refer the reader to Appendix $\mathrm{A}$ for the formal definition of incrementing counter automata.

In short, an incrementing $l$-counter automaton over $\Sigma$ is an automaton with $l$ counters, operates on words over $\Sigma$, and the value in each counter is allowed to erroneously increase, hence, the name incrementing.

A configuration is a tuple $(q, \sigma, \boldsymbol{v})$ where $q$ is a state, $\sigma$ is the current symbol read and $\boldsymbol{v}:\{1, \ldots, l\} \rightarrow \mathbb{N}$, where $\boldsymbol{v}(i)$ denotes the value stored in counter $i$.

Now a configuration $(q, \boldsymbol{v})$ can be encoded as a $\left(Q \cup \Sigma \cup\left\{c_{1}, \ldots, c_{l}\right\}\right)$-data word as follows.

$$
\left(\begin{array}{c}
q \\
d_{1}
\end{array}\right)\left(\begin{array}{c}
\sigma \\
d_{2}
\end{array}\right)\left(\begin{array}{c}
c_{1} \\
a_{1,1}
\end{array}\right) \cdots\left(\begin{array}{c}
c_{1} \\
a_{1,}, \boldsymbol{v}(1)
\end{array}\right) \cdots\left(\begin{array}{c}
c_{l} \\
a_{l, 1}
\end{array}\right) \cdots\left(\begin{array}{c}
c_{l} \\
a_{l, \boldsymbol{v}(l)}
\end{array}\right) .
$$

where the symbols $a_{1,1}, \ldots, a_{l, \boldsymbol{v}(l)}$ are pairwise different. The labels $c_{1}, \ldots, c_{l}$ are used as pointers that the current data value is part of the encoding of the counters $\boldsymbol{v}(1), \ldots, \boldsymbol{v}(l)$, respectively. 
Since the automaton allows for erroneous increment of values in each counter, we can check the validity of the application of each transition, like in Examples 9, 10 and 11.

Now we are going to show the NP-completeness of the labelling problem. It is by a reduction from graph 3-colorability problem.

Given an undirected graph $G=(V, E)$, let $V=\{1, \ldots, n\}$ and $E=$ $\left\{\left(i_{1}, j_{1}\right), \ldots,\left(i_{m}, j_{m}\right)\right\}$. We can take $i_{1} j_{1} \cdots i_{m} j_{m}$ as the sequence of data values. Then, we construct a weak 2-PA $\mathcal{A}$ over the alphabet $\Sigma=\left\{\vartheta_{R}, \vartheta_{G}, \vartheta_{B}\right\}$ that accepts data words of even length in which the following hold.

- For all odd position $x$, the label on position $x$ is different from the label on position $x+1$.

- For every two positions $x$ and $y$, if they have the same data value, then they have the same label.

Thus, the graph $G$ is 3-colorable if and only if there exists $\sigma_{1} \cdots \sigma_{2 m} \in$ $\left\{\vartheta_{R}, \vartheta_{G}, \vartheta_{B}\right\}^{*}$ such that

$$
\left(\begin{array}{c}
\sigma_{1} \\
i_{1}
\end{array}\right)\left(\begin{array}{c}
\sigma_{2} \\
j_{1}
\end{array}\right) \cdots\left(\begin{array}{c}
\sigma_{2 m-1} \\
i_{m}
\end{array}\right)\left(\begin{array}{c}
\sigma_{2 m} \\
j_{m}
\end{array}\right) \in L(\mathcal{A}),
$$

and the NP-completeness of the labeling problem follows.

The NP-completeness of data value membership problem can established in a similar spirit. The reduction is from the following variant of graph 3colorability, called 3-colorability with constraint. Given a graph $G=(V, E)$ and three integers $n_{r}, n_{g}, n_{b}$ in unary form, can the graph $G$ be colored with the colors $R, G$ and $B$ such that the numbers of vertices colored with $R, G$ and $B$ are $n_{r}, n_{g}$ and $n_{b}$, respectively?

The polynomial time reduction to data value membership problem is as follows. Let $V=\{1, \ldots, n\}$ and $E=\left\{\left(i_{1}, j_{1}\right), \ldots,\left(i_{m}, j_{m}\right)\right\}$.

We define $\Sigma=\left\{\vartheta_{R}, \vartheta_{G}, \vartheta_{B}, \nu_{1}, \ldots, \nu_{n}\right\}$. We take

$$
\nu_{i_{1}} \nu_{j_{1}} \cdots \nu_{i_{m}} \nu_{j_{m}} \underbrace{\vartheta_{R} \cdots \vartheta_{R}}_{n_{r} \text { times }} \underbrace{\vartheta_{G} \cdots \vartheta_{G}}_{n_{g} \text { times }} \underbrace{\vartheta_{B} \cdots \vartheta_{B}}_{n_{b} \text { times }}
$$

as the sequence of finite labels.

Then, we construct a weak 2-PA over $\Sigma$ that accepts data words of the form

$\left(\begin{array}{c}\nu_{i_{1}} \\ c_{1}\end{array}\right)\left(\begin{array}{c}\nu_{j_{1}} \\ d_{1}\end{array}\right) \cdots\left(\begin{array}{c}\nu_{i_{m}} \\ c_{m}\end{array}\right)\left(\begin{array}{c}\nu_{j_{m}} \\ d_{m}\end{array}\right)\left(\begin{array}{c}\vartheta_{R} \\ a_{1}\end{array}\right) \cdots\left(\begin{array}{c}\vartheta_{R} \\ a_{n_{r}}\end{array}\right)\left(\begin{array}{c}\vartheta_{G} \\ a_{1}^{\prime}\end{array}\right) \cdots\left(\begin{array}{c}\vartheta_{G} \\ a_{n_{g}}^{\prime}\end{array}\right)\left(\begin{array}{c}\vartheta_{B} \\ a_{1}^{\prime \prime}\end{array}\right) \cdots\left(\begin{array}{c}\vartheta_{B} \\ a_{n_{b}}^{\prime \prime}\end{array}\right)$ 
where

- $\nu_{i_{1}}, \nu_{j_{1}}, \ldots, \nu_{i_{m}}, \nu_{j_{m}} \in\left\{\nu_{1}, \ldots, \nu_{n}\right\}$

- in the sub-word $\left(\begin{array}{c}\nu_{i_{1}} \\ c_{1}\end{array}\right)\left(\begin{array}{c}\nu_{j_{1}} \\ d_{1}\end{array}\right) \cdots\left(\begin{array}{c}\nu_{i_{m}} \\ c_{m}\end{array}\right)\left(\begin{array}{c}\nu_{j_{m}} \\ d_{m}\end{array}\right)$, every two positions with the same labels have the same data value, see Example 4,

- the data values $a_{1}, \ldots, a_{n_{r}}, a_{1}^{\prime}, \ldots, a_{n_{g}}^{\prime}, a_{1}^{\prime \prime}, \ldots, a_{n_{b}}^{\prime \prime}$ are pairwise different;

- For each $i=1, \ldots, m$, the data values $c_{i}, d_{i}$ appear among $a_{1}, \ldots, a_{n_{r}}$, $a_{1}^{\prime}, \ldots, a_{n_{g}}^{\prime}, a_{1}^{\prime \prime}, \ldots, a_{n_{b}}^{\prime \prime}$ such that the following holds:

- if $c_{i}$ appears among $a_{1}, \ldots, a_{n_{r}}$, then $d_{i}$ appears among $a_{1}^{\prime}, \ldots, a_{n_{g}}^{\prime}$ or $a_{1}^{\prime \prime}, \ldots, a_{n_{b}}^{\prime \prime}$;

- if $c_{i}$ appears among $a_{1}^{\prime}, \ldots, a_{n_{g}}^{\prime}$, then $d_{i}$ appears either among $a_{1}, \ldots, a_{n_{r}}$ or $a_{1}^{\prime \prime}, \ldots, a_{n_{b}}^{\prime \prime}$; and

- if $c_{i}$ appears among $a_{1}^{\prime \prime}, \ldots, a_{n_{b}}^{\prime \prime}$, then $d_{i}$ appears among $a_{1}, \ldots, a_{n_{r}}$ or $a_{1}^{\prime}, \ldots, a_{n_{g}}^{\prime}$.

Note that we can store the integers $r, g, b$ and $m$ in the internal states $\mathcal{A}$, thus, enable $\mathcal{A}$ to "count" up to $n_{r}, n_{g}, n_{b}$ and $m$. We have each state for the numbers $1, \ldots, n_{r}, 1, \ldots, n_{g}, 1, \ldots, n_{b}$ and $1, \ldots, m$. Furthermore, the unary form of $n_{r}, n_{g}$ and $n_{b}$ is crucial here to ensure that the number of the states of $\mathcal{A}$ is still polynomial in the length of the input.

Now the graph $G$ is 3-colorable with constraint if and only if there exits $c_{1} d_{1} \cdots c_{m} d_{m} a_{1} \cdots a_{n_{r}} a_{1}^{\prime} \cdots a_{n_{g}}^{\prime} a_{1}^{\prime \prime} \cdots a_{n_{b}}^{\prime \prime}$ such that

$\left(\begin{array}{c}\nu_{i_{1}} \\ c_{1}\end{array}\right)\left(\begin{array}{c}\nu_{j_{1}} \\ d_{1}\end{array}\right) \cdots\left(\begin{array}{c}\nu_{i_{m}} \\ c_{m}\end{array}\right)\left(\begin{array}{c}\nu_{j_{m}} \\ d_{m}\end{array}\right)\left(\begin{array}{c}\vartheta_{R} \\ a_{1}\end{array}\right) \cdots\left(\begin{array}{c}\vartheta_{R} \\ a_{n_{r}}\end{array}\right)\left(\begin{array}{c}\vartheta_{G} \\ a_{1}^{\prime}\end{array}\right) \cdots\left(\begin{array}{c}\vartheta_{G} \\ a_{n_{g}}^{\prime}\end{array}\right)\left(\begin{array}{c}\vartheta_{B} \\ a_{1}^{\prime \prime}\end{array}\right) \cdots\left(\begin{array}{c}\vartheta_{B} \\ a_{n_{b}}^{\prime \prime}\end{array}\right)$

is accepted by $\mathcal{A}$, and the NP-completeness of data value membership problem follows.

\section{Top view weak $k$-PA}

In this section we are going to restrict the definition of weak $k$-PA so that its emptiness problem becomes decidable. Roughly speaking, top view weak PA are weak PA where the equality test is performed only between the data values seen by the last and the second last placed pebbles. That is, if pebble $i$ 
is the head pebble, then it can only compare the data value it reads with the data value read by pebble $(i+1)$. It is not allowed to compare its data value with those read by pebble $i+2, \ldots, k$.

Formally, the transitions of top view weak $k$-PA $\mathcal{A}=\left\langle Q, q_{0}, \mu, F\right\rangle$ are of the form

$$
(i, \sigma, V, q) \rightarrow\left(q^{\prime}, \text { act }\right)
$$

where $V$ is either $\emptyset$ or $\{i+1\}$.

The definition of top view weak $k$-PA is defined by setting

$$
V= \begin{cases}\emptyset, & \text { if } a_{\theta(i+1)} \neq a_{\theta(i)} \\ \{i+1\}, & \text { if } a_{\theta(i+1)}=a_{\theta(i)}\end{cases}
$$

in the definition of transition relation in Subsection 2.1. Note that top view weak 2-PA are just the same as weak 2-PA. We can also define the alternating version of top view weak $k$-PA. However, just like in the case of weak $k$-PA, alternating, nondeterministic and deterministic top view weak $k$-PA have the same recognition power.

Theorem 13 For every top view weak $k$-PA $\mathcal{A}$, there is a one-way alternating 1-RA $\mathcal{A}^{\prime}$ such that $L\left(\mathcal{A}^{\prime}\right)=L(\mathcal{A})$. Moreover, the construction of $\mathcal{A}^{\prime}$ is effective.

Proof. The proof is a straightforward generalization of the proof of Theorem 7. Each placement of a pebble is simulated by "Guess-Split-Verify" procedure. Since each pebble $i$ can only compare its data value with the one seen by pebble $i+1, \mathcal{A}^{\prime}$ does not need to store the data values seen by pebble $i+2, \ldots, k$. It only need to store the data value seen by pebble $i+1$, thus, one register suffices.

Following Theorem 21, we immediately obtain the decidability of the emptiness problem for top view weak $k$-PA.

Corollary 14 The emptiness problem for top view weak $k$-PA is decidable.

Remark 15 Since the emptiness problem for ordinary 2-PA and for weak 3-PA is already undecidable (See Theorem [6 and [10, Theorem 4]), it seems that top view weak PA is a tight boundary of a subclass of PA languages for which the emptiness problem is decidable. 
Theorem 16 For every sentence $\psi \in L T L^{\downarrow}(\Sigma, X, U)$, there exists a top view weak $k$-PA $\mathcal{A}_{\psi}$, where $k=$ far $(\psi)+1$, such that $L\left(\mathcal{A}_{\psi}\right)=L(\psi)$.

Proof. Let $\psi$ be an $\operatorname{LTL}^{\downarrow}(\Sigma, \mathrm{X}, \mathrm{U})$ sentence. We construct an alternating top view weak $k$-PA $\mathcal{A}_{\psi}$, where $k=\mathrm{fgr}(\psi)+1$ such that given a data word $w$, the automaton $\mathcal{A}_{\psi}$ checks whether $w, 1 \models \psi$. $\mathcal{A}_{\psi}$ accepts if it is so. Otherwise, it rejects.

Intuitively, the computation of $w, 1 \models \psi$ is done recursively as follows. The automaton $\mathcal{A}_{\psi}$ "consists of" the automata $\mathcal{A}_{\varphi}$ for all sub-formula $\varphi$ of $\psi$, including $\mathcal{A}_{\epsilon}$ to represent the empty formula $\epsilon$.

- The automaton $\mathcal{A}_{\epsilon}$ accepts every data words.

- If $\psi=\sigma \varphi$, then check whether the current label is $\sigma$. If it is not, then $\mathcal{A}$ rejects immediately. Otherwise, $\mathcal{A}_{\psi}$ proceeds to run $\mathcal{A}_{\varphi}$.

- If $\psi=\varphi \vee \varphi^{\prime}$, then $\mathcal{A}_{\psi}$ nondeterministically chooses one of $\mathcal{A}_{\varphi}$ or $\mathcal{A}_{\varphi^{\prime}}$ and proceeds to run one of them.

- If $\psi=\varphi \wedge \varphi^{\prime}$, then $\mathcal{A}_{\psi}$ splits its computation (by conjunctive branching) into two and proceed to run both of $\mathcal{A}_{\varphi}$ and $\mathcal{A}_{\varphi^{\prime}}$.

- If $\psi=\mathrm{X} \varphi$, then $\mathcal{A}_{\psi}$ moves to the right one step. If it reads the rightend marker, then the automaton rejects immediately. Otherwise, it proceeds to run $\mathcal{A}_{\varphi}$.

- If $\psi=\uparrow \varphi$, then $\mathcal{A}_{\psi}$ checks whether the data value seen by its head pebble is the same as the one seen by the second last placed pebble. If it is not the same, then it rejects immediately. Otherwise, it proceeds to run $\mathcal{A}_{\varphi}$.

- If $\psi=\downarrow \varphi$, then $\mathcal{A}_{\psi}$ places a new pebble and proceeds to run $\mathcal{A}_{\varphi}$.

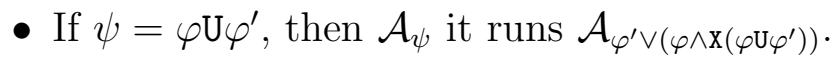

- If $\psi=\neg \varphi$, then $\mathcal{A}_{\psi}$ runs $\mathcal{A}_{\varphi}$. If $\mathcal{A}_{\varphi}$ accepts, then $\mathcal{A}_{\psi}$ rejects. Otherwise, $\mathcal{A}_{\psi}$ accepts.

Note that since $\operatorname{fgr}(\varphi)=k$, on each computation path then the automaton $\mathcal{A}_{\psi}$ only needs to place the pebble $k$ times, thus, $\mathcal{A}_{\psi}$ requires only $k+1$. It is a straight forward induction to show that $L\left(\mathcal{A}_{\psi}\right)=L(\psi)$. 
Our next results deals with the expressive power of $\operatorname{LTL}_{1}^{\downarrow}(\Sigma, \mathrm{X}, \mathrm{U})$ based on the freeze quantifier rank. It is an analog of the classical hierarchy of first order logic based on the ordinary quantifier rank. We start by defining an $\operatorname{LTL}_{1}^{\downarrow}(\Sigma, \mathrm{X}, \mathrm{U})$ sentence for the language $\mathcal{R}_{m}^{+}$defined in Subsection 2.2 .

Lemma 17 For each $k=1,2,3, \ldots$, there exists a sentence $\psi_{k}$ in $L T L_{1}^{\downarrow}(\Sigma, X, U)$ such that $L\left(\psi_{k}\right)=\mathcal{R}_{k}^{+}$and

- $\operatorname{far}\left(\psi_{1}\right)=1$; and

- $\operatorname{fqr}\left(\psi_{k}\right)=k-1$, when $k \geq 2$.

Proof. First, we define a formula $\varphi_{k}$ such that $\operatorname{fgr}\left(\varphi_{k}\right)=k-1$ and for every data word $w=\left(\begin{array}{c}\sigma \\ d_{1}\end{array}\right) \cdots\left(\begin{array}{c}\sigma \\ d_{n}\end{array}\right)$, for every $i=1, \ldots, n$,

$$
w, i \models_{d_{i}} \varphi_{k} \text { if and only if }\left(\begin{array}{c}
\sigma \\
d_{i}
\end{array}\right) \cdots\left(\begin{array}{c}
\sigma \\
d_{n}
\end{array}\right) \in \mathcal{R}_{k}^{+} .
$$

We construct $\varphi_{k}$ inductively as follows.

- $\varphi_{1}:=\mathrm{X}(\neg \uparrow) \wedge \neg(\mathrm{X}$ True $)$.

- For each $k=1,2,3, \ldots$,

$$
\varphi_{k+1}:=\mathrm{X}(\neg \uparrow) \wedge \mathrm{X}\left(\downarrow \mathrm{X}\left((\neg \uparrow) \mathrm{U}\left(\uparrow \wedge \varphi_{k}\right)\right)\right)
$$

Note that since $\operatorname{fqr}\left(\varphi_{1}\right)=0$, then for each $k=1,2, \ldots, \operatorname{fqr}\left(\varphi_{k}\right)=k-1$.

Assuming first that $\varphi_{k}$ satisfies the property in Equation [3, the desired sentence $\psi_{k}$ is defined as follows.

- $\psi_{1}:=\downarrow(\mathrm{X}(\neg \uparrow) \wedge \neg(\mathrm{X}$ True $))$.

- For each $k=2,3, \ldots$,

$$
\psi_{k}:=\downarrow(\mathrm{X}(\neg \uparrow)) \wedge \mathrm{X}\left(\downarrow \mathrm{X}\left((\neg \uparrow) \mathrm{U}\left(\uparrow \wedge \varphi_{k-1}\right)\right)\right)
$$

Since $\operatorname{fqr}\left(\varphi_{k-1}\right)=k-2$, then $\operatorname{fqr}\left(\psi_{k}\right)=k-1$.

Now we want to show that the formula $\varphi_{k}$ satisfies Equation 3 . The proof is by induction on $k$. The base case, $k=1$, is trivial. Suppose, for the induction hypothesis, the formula $\varphi_{k}$ satisfies Equation 3 , 
The induction step is as follows. Let $w=\left(\begin{array}{c}\sigma \\ d_{1}\end{array}\right) \cdots\left(\begin{array}{c}\sigma \\ d_{n}\end{array}\right)$. We have the following chain of application of the semantics of LTL.

$$
\begin{aligned}
& w, i \quad \models_{d_{i}} \varphi_{k+1} \\
& \Uparrow \\
& w, i \quad \models_{d_{i}} \mathrm{X}(\neg \uparrow) \wedge \mathrm{X}\left(\downarrow \mathrm{X}\left((\neg \uparrow) \mathrm{U}\left(\uparrow \wedge \varphi_{k}\right)\right)\right) \\
& \Uparrow \\
& w, i \quad \models_{d_{i}} \quad \mathrm{X}(\neg \uparrow) \quad \text { and } \quad w, i \quad \models_{d_{i}} \quad \mathrm{X}\left(\downarrow \mathrm{X}\left((\neg \uparrow) \mathrm{U}\left(\uparrow \wedge \varphi_{k}\right)\right)\right)
\end{aligned}
$$

For the first part, we have

$$
w, i \models_{d_{i}} \mathrm{X}(\neg \uparrow) \text { if and only if } d_{i} \neq d_{i+1}
$$

Now we evaluate the second part.

$$
\begin{array}{rcr}
w, i & \models_{d_{i}} & \mathrm{X}\left(\downarrow \mathrm{X}\left((\neg \uparrow) \mathrm{U}\left(\uparrow \wedge \varphi_{k}\right)\right)\right) \\
& \mathbb{\Downarrow} & \\
w, i+1 & \models_{d_{i}} & \left(\downarrow \mathrm{X}\left((\neg \uparrow) \mathrm{U}\left(\uparrow \wedge \varphi_{k}\right)\right)\right) \\
& \mathbb{\Downarrow} & \\
w, i+1 & \models_{d_{i+1}} & \mathrm{X}\left((\neg \uparrow) \mathrm{U}\left(\uparrow \wedge \varphi_{k}\right)\right) \\
& \mathbb{\Downarrow} & \\
w, i+2 & \models_{d_{i+1}} & (\neg \uparrow) \mathrm{U}\left(\uparrow \wedge \varphi_{k}\right)
\end{array}
$$

Equation 5 holds if and only if there exists $j$ such that $i+2 \leq j$ and

1. $w, j \models d_{i+1} \uparrow \wedge \varphi_{k}$,

2. $w, j^{\prime} \models_{d_{i+1}} \neg \uparrow$, for each $j^{\prime}=i+1, \ldots, j-1$.

By the semantics of LTL and the induction hypothesis, Clause 1 holds if and only if $d_{j}=d_{i+1}$ and $\left(\begin{array}{c}\sigma \\ d_{j}\end{array}\right) \cdots\left(\begin{array}{c}\sigma \\ d_{n}\end{array}\right) \in \mathcal{R}_{k}^{+}$. The meaning of Clause 2 is $d_{j}^{\prime} \neq d_{i+1}$, for each $j^{\prime}=i+1, \ldots, j-1$. Both clauses, together with Equation 4, means that $\left(\begin{array}{c}\sigma \\ d_{i}\end{array}\right) \cdots\left(\begin{array}{c}\sigma \\ d_{n}\end{array}\right) \in \mathcal{R}_{k+1}^{+}$. This completes the induction hypothesis.

Lemma 18 For each $k=1,2, \ldots$, the language $\mathcal{R}_{k+1}^{+}$is not expressible by a sentence in $\operatorname{LTL}_{1}^{\downarrow}(\Sigma, X, U)$ of freeze quantifier rank $k-1$. 
Proof. By Theorem $5, \mathcal{R}_{k+1}^{+}$is not accepted by weak $k$-PA, thus, it is also not accepted by top-view $k$-PA. Then, by Theorem $16, \mathcal{R}_{k+1}^{+}$is not expressible by $\operatorname{LTL}^{\downarrow}(\Sigma, \mathrm{X}, \mathrm{U})$ sentence of freeze quantifier rank $k-1$.

Combining both Lemmas 17 and 18, we obtain the following strict hierarchy of $\operatorname{LTL}^{\downarrow}(\Sigma, \mathrm{X}, \mathrm{U})$ based on its freeze quantifier rank.

Theorem 19 For each $k=1,2, \ldots$, the class of sentences in $\operatorname{LTL}^{\downarrow}(\Sigma, X, U)$ of freeze quantifier rank $k+1$ is strictly more expressive than those of freeze quantifier rank $k$.

\section{Top view weak $k$-PA}

In this section we are going to define top view weak PA. Roughly speaking, top view weak PA are weak PA where the equality test is performed only between the data values seen by the last and the second last placed pebbles. That is, if pebble $i$ is the head pebble, then it can only compare the data value it reads with the data value read by pebble $(i+1)$. It is not allowed to compare its data value with those read by pebble $(i+2),(i+3), \ldots, k$.

Formally, top view weak $k$-PA is a tuple $\mathcal{A}=\left\langle\Sigma, Q, q_{0}, \mu, F\right\rangle$ where $Q, q_{0}, F$ are as usual and $\mu$ consists of transitions of the form: $(i, \sigma, V, q) \rightarrow$ $\left(q^{\prime}\right.$, act), where $V$ is either $\emptyset$ or $\{i+1\}$.

The criteria for the application of transitions of top view weak $k$-PA is defined by setting

$$
V= \begin{cases}\emptyset, & \text { if } a_{\theta(i+1)} \neq a_{\theta(i)} \\ \{i+1\}, & \text { if } a_{\theta(i+1)}=a_{\theta(i)}\end{cases}
$$

in the definition of transition relation in Subsection 2.1. Note that top view weak 2-PA and weak 2-PA are the same.

Remark 20 We can also define the alternating version of top view weak $k$-PA. However, just like in the case of weak $k$-PA, alternating, nondeterministic and deterministic top view weak $k$-PA have the same recognition power. Furthermore, by using the same proof presented in Section 4, it is straightforward to show that the emptiness problem, the labelling problem, and the data value membership problem have the same complexity lower bound for top view weak $k$-PA, for each $k=2,3, \ldots$

The following theorem is a stronger version of Theorem 7 . 
Theorem 21 For every top view weak $k$-PA $\mathcal{A}$, there is a one-way alternating 1-RA $\mathcal{A}^{\prime}$ such that $L\left(\mathcal{A}^{\prime}\right)=L(\mathcal{A})$. Moreover, the construction of $\mathcal{A}^{\prime}$ is effective.

Proof. The proof is a straightforward generalization of the proof of Theorem 7 . Each placement of a pebble is simulated by "Guess-Split-Verify" procedure. Since each pebble $i$ can only compare its data value with the one seen by pebble $(i+1), \mathcal{A}^{\prime}$ does not need to store the data values seen by pebbles $(i+2), \ldots, k$. It only needs to store the data value seen by pebble $(i+1)$, thus, one register is sufficient for the simulation.

Following Theorem [21, we immediately obtain the decidability of the emptiness problem for top view weak $k$-PA.

Corollary 22 The emptiness problem for top view weak $k$-PA is decidable.

Since the emptiness problem for ordinary 2-PA (See [10, Theorem 4]) and for weak 3-PA is already undecidable, it seems that top view weak PA is a tight boundary of a subclass of PA languages for which the emptiness problem is decidable.

Remark 23 In [16] it is shown that for every sentence $\psi \in \operatorname{LTL}_{1}^{\downarrow}(\Sigma, \mathrm{X}, \mathrm{U})$, there exists a weak $k$-PA $\mathcal{A}_{\psi}$, where $k=\mathrm{fgr}(\psi)+1$, such that $L\left(\mathcal{A}_{\psi}\right)=L(\psi)$. We remark that the proof actually shows that the automaton $\mathcal{A}_{\psi}$ is top view weak $k$-PA. Thus, it shows that the class of top view weak $k$-PA languages contains the languages definable by $\operatorname{LTL}_{1}^{\downarrow}(\Sigma, \mathrm{X}, \mathrm{U})$.

\section{Top view weak PA with unbounded num- ber of pebbles}

This section contains our quick observation on top view weak PA. We note that the finiteness of the number of pebbles for top view weak PA is not necessary. In fact, we can just define top view weak PA with unbounded number of pebbles, which we call top view weak unbounded PA.

We elaborate on it in the following paragraphs. Let $\mathcal{A}=\left\langle\Sigma, Q, q_{0}, \mu, F\right\rangle$ be top view weak unbounded PA. The pebbles are numbered with the numbers $1,2,3, \ldots$. The automaton $\mathcal{A}$ starts the computation with only pebble 1 
on the input word. The transitions are of the form: $(\sigma, \chi, q) \rightarrow(p$, act $)$, where $\chi \in\{0,1\}$ and $\sigma, q, p$, act are as in the ordinary weak PA.

Let $w=\left(\begin{array}{c}\sigma_{1} \\ a_{1}\end{array}\right) \cdots\left(\begin{array}{c}\sigma_{n} \\ a_{n}\end{array}\right)$ be an input word. A configuration of $\mathcal{A}$ on $\triangleleft w \triangleright$ is a triple $[i, q, \theta]$, where $i \in \mathbb{N}, q \in Q$, and $\theta: \mathbb{N} \rightarrow\{0,1, \ldots, n, n+1\}$. The initial configuration is $\left[1, q_{0}, \theta_{0}\right]$, where $\theta_{0}(1)=0$. The accepting configurations are defined similarly as in ordinary weak PA.

A transition $(\sigma, \chi, p) \rightarrow \beta$ applies to a configuration $[i, q, \theta]$, if

(1) $p=q$, and $\sigma_{\theta(i)}=\sigma$,

(2) $\chi=1$ if $a_{\theta(i-1)}=a_{\theta(i)}$, and $\chi=0$ if $a_{\theta(i-1)} \neq a_{\theta(i)}$,

Similarly, the transition relation $\vdash$ can be defined as follows: $[i, q, \theta] \vdash_{\mathcal{A}}$ $\left[i^{\prime}, q^{\prime}, \theta^{\prime}\right]$, if there is a transition $\alpha \rightarrow(p$, act $) \in \mu$ that applies to $[i, q, \theta]$ such that $q^{\prime}=p$, for all $j<i, \theta^{\prime}(j)=\theta(j)$, and

- if act $=$ right, then $i^{\prime}=i$ and $\theta^{\prime}(i)=\theta(i)+1$,

- if act $=$ lift-pebble, then $i^{\prime}=i-1$

- if act $=$ place-pebble, then $i^{\prime}=i+1, \theta^{\prime}(i+1)=\theta^{\prime}(i)=\theta(i)$.

The acceptance criteria can be defined similarly.

It is straightforward to show that 1-way deterministic 1-RA can be simulated by top view weak unbounded PA. Each time the register automaton change the content of the register, the top view weak unbounded PA places a new pebble.

Furthermore, top view weak unbounded PA can be simulated by 1-way alternating 1-RA. Each time a pebble is placed, the register automaton performs "Guess-Split-Verify" procedure described in Section 3. Thus, the emptiness problem for top view unbounded weak PA is still decidable.

\section{Concluding remark}

In this paper we study pebble automata for data languages. In particular, we establish a fragment of PA languages for which the emptiness problem is decidable, the so called top view weak PA. As shown in this paper, top view weak PA inherit some nice properties mentioned in Section 1 .

1. Expressiveness: Top view weak PA strictly contain the languages expressible by $\operatorname{LTL}_{1}^{\downarrow}(\Sigma, \mathrm{X}, \mathrm{U})$. 
2. Decidability: The emptiness problem is decidable.

3. Efficiency: The model checking problem, that is, testing whether a given string of length $n$ is accepted by a specific deterministic top view weak $k$-PA can be solved in $O\left(n^{k}\right)$ computation time.

4. Closure properties: Top view weak $k$-PA languages are closed under all boolean operations.

5. Robustness: Alternation and nondeterminism do not add expressive power to top view weak $k$-PA languages.

There are still lots of work to be done. In order to be applicable in program verification and XML settings, the model should work be infinite strings and unranked trees, respectively. Thus, the question remains whether it is possible to extend top view weak PA to the settings of infinite strings and unranked trees, while still preserving the five properties mentioned above.

Acknowledgment. The author would like to thank Michael Kaminski for his invaluable directions and guidance related to this paper and for pointing out the notion of unbounded pebble automata.

\section{References}

[1] Parosh Aziz Abdulla, Bengt Jonsson, Marcus Nilsson, and Mayank Saksena. A survey of regular model checking. In Proceedings of the 15th International Conference on Concurrency Theory (CONCUR) 2004, volume 3170 of Lecture Notes in Computer Science, pages 35-48. Springer, 2004.

[2] Marcelo Arenas, Wenfei Fan, and Leonid Libkin. Consistency of XML specifications. In Inconsistency Tolerance [Dagstuhl Seminar], volume 3300 of Lecture Notes in Computer Science, pages 15-41. Springer, 2005.

[3] Henrik Björklund and Thomas Schwentick. On notions of regularity for data languages. In Proceedings of the 16th International Symposium on Fundamentals of Computation Theory, FCT 200\%, volume 4639 of Lecture Notes in Computer Science, pages 88-99. Springer, 2007. 
[4] Mikolaj Bojanczyk, Anca Muscholl, Thomas Schwentick, Luc Segoufin, and Claire David. Two-variable logic on words with data. In Proceedings of the 21th IEEE Symposium on Logic in Computer Science (LICS 2006), pages 7-16. IEEE Computer Society, 2006.

[5] Mikolaj Bojanczyk, Mathias Samuelides, Thomas Schwentick, and Luc Segoufin. Expressive power of pebble automata. In Part I of the Proceedings of Automata, Languages and Programming, 33rd International Colloquium, ICALP 2006, volume 4051 of Lecture Notes in Computer Science, pages 157-168. Springer, 2006.

[6] Stéphane Demri and Ranko Lazic. Ltl with the freeze quantifier and register automata. In Proceedings of the 21th IEEE Symposium on Logic in Computer Science (LICS) 2006, pages 17-26. IEEE Computer Society, 2006.

[7] Allen Emerson and Kedar Namjoshi. Reasoning about rings. In Proceedings of the 22nd ACM SIGPLAN-SIGACT Symposium on Principles of Programming Languages (POPL) 1995, pages 85-94, 1995.

[8] John Hopcroft and Jeffrey Ullman. Introduction to Automata Theory, Languages and Computation. Addison-Wesley, 1979.

[9] Michael Kaminski and Nissim Francez. Finite-memory automata. Theoretical Computer Science, 134(2):329-363, 1994.

[10] Michael Kaminski and Tony Tan. A note on two-pebble automata over infinite alphabets. Technical Report CS2009-02, Department of Computer Science, Technion - Israel Institute of Technology, 2009. Can be found in http://www.cs.technion.ac.il/users/wwwb/cgi-bin/tr-list.cgi/2009/CS.

[11] Richard Mayr. Undecidable problems in unreliable computations. Theoretical Computer Science, 297(1-3):337-354, 2003.

[12] Frank Neven. Automata, logic, and xml. In Proceedings of the 11th Annual Conference of the EACSL Computer Science Logic (CSL) 2002, volume 2471 of Lecture Notes in Computer Science, pages 2-26. Springer, 2002. 
[13] Frank Neven, Thomas Schwentick, and Victor Vianu. Finite state machines for strings over infinite alphabets. ACM Transactions on Computational Logic, 5(3):403-435, 2004.

[14] Philippe Schnoebelen. Verifying lossy channel systems has nonprimitive recursive complexity. Information Processing Letters, 83(5):251261, 2002.

[15] Luc Segoufin. Automata and logics for words and trees over an infinite alphabet. In Proceedings of the 20th International Workshop/15th Annual Conference of the EACSL Computer Science Logic, CSL 2006, pages 41-57, 2006.

[16] Tony Tan. Graph reachability and pebble automata over infinite alphabets. In Proceedings of the 24th IEEE Symposium on Logic in Computer Science (LICS) 2009, pages 157-166. IEEE Computer Society, 2009.

[17] Tony Tan. On pebble automata for data languages with decidable emptiness problem. Technical Report CS-2009-05, Department of Computer Science, Technion - Israel Institute of Technology, 2009. Can be found in http://www.cs.technion.ac.il/users/wwwb/cgi-bin/tr-list.cgi/2009/CS.

\section{A Counter Automata}

A Minsky $k$-counter automata (CA), with $\epsilon$-transitions and zero testing, is a tuple $\mathcal{A}=\left\langle\Sigma, Q, q_{0}, \delta, F\right\rangle$, where

- $\Sigma$ is a finite alphabet;

- $Q$ is a finite set of states;

- $q_{0}$ is the initial state;

- $\delta \subseteq Q \times(\Sigma \cup\{\epsilon\}) \times L \times Q$ is a transition relation over the instruction set $L=\{$ inc, dec, if $z\} \times\{1, \ldots, k\}$;

- $F \subseteq Q$ is the set of accepting set, such that $q^{\prime} \notin F$ whenever $\left(q, \epsilon, l, q^{\prime}\right) \in$ $\delta$. 
Given a word $w=\sigma_{1} \cdots \sigma_{n} \in \Sigma^{*}$, a configuration of $\mathcal{A}$ is a triple $[i, q, \boldsymbol{v}]$ where $0 \leq i \leq n, q \in Q$ and a counter valuation $\boldsymbol{v}:\{1, \ldots, k\} \rightarrow \mathbb{N}$, where $\mathbb{N}$ is the set of natural number $\{1,2,3, \ldots\}$.

The initial configuration is $\left[0, q_{0}, \boldsymbol{v}_{0}\right]$ where $\boldsymbol{v}_{0}(j)=0$ for each $j=1, \ldots, k$. The run of $\mathcal{A}$ on $w$ is a sequence $\left[0, q_{0}, \boldsymbol{v}_{0}\right],\left[1, q_{1}, \boldsymbol{v}_{1}\right], \ldots,\left[n, q_{n}, \boldsymbol{v}_{n}\right]$ where for each $i=0, \ldots, n-1$, there exists a transition $\left(q_{i}, \sigma_{i+1}, l, q_{i+1}\right) \in \delta$ and

- if $l=($ inc, $j)$ for some $j=1 \ldots, k$, then $\boldsymbol{v}_{i+1}(j)=\boldsymbol{v}_{i}(j)+1$ and for all other $j^{\prime} \neq j, \boldsymbol{v}_{i+1}\left(j^{\prime}\right)=\boldsymbol{v}_{i}\left(j^{\prime}\right)$.

- if $l=(\operatorname{dec}, j)$ for some $j=1 \ldots, k$, then $\boldsymbol{v} i(j)>0$ and $\boldsymbol{v}_{i+1}(j)=$ $\boldsymbol{v}_{i}(j)-1$ and for all other $j^{\prime} \neq j, \boldsymbol{v}_{i+1}\left(j^{\prime}\right)=\boldsymbol{v}_{i}\left(j^{\prime}\right)$.

- if $l=($ if $z, j)$ for some $j=1 \ldots, k$, then $\boldsymbol{v}_{i}(j)=0$ and $\boldsymbol{v}_{i+1}=\boldsymbol{v}_{i}$.

The word $w$ is accepted by $\mathcal{A}$ if $q_{n} \in F$. As usual, we denote by $L(\mathcal{A})$ the set of all words over $\Sigma$ accepted by $\mathcal{A}$.

We say that the automaton $\mathcal{A}$ is incrementing if its counters may erroneously increase at any time. More precisely, The run of an incrementing $\mathcal{A}$ on $w$ is a sequence of configurations $\left[0, q_{0}, \boldsymbol{v}_{0}\right],\left[1, q_{1}, \boldsymbol{v}_{1}\right], \ldots,\left[n, q_{n}, \boldsymbol{v}_{n}\right]$ where for each $i=0, \ldots, n-1$, there exists a transition $\left(q_{i}, \sigma_{i+1}, l, q_{i+1}\right) \in \delta$ and

- if $l=\left(\right.$ inc, $j$ ) for some $j=1 \ldots, k$, then $\boldsymbol{v}_{i+1}(j) \geq \boldsymbol{v}_{i}(j)+1$ and for all other $j^{\prime} \neq j, \boldsymbol{v}_{i+1}\left(j^{\prime}\right) \geq \boldsymbol{v}_{i}\left(j^{\prime}\right)$.

- if $l=(\operatorname{dec}, j)$ for some $j=1 \ldots, k$, then $\boldsymbol{v} i(j)>0$ and $\boldsymbol{v}_{i+1}(j) \geq$ $\boldsymbol{v}_{i}(j)-1$ and for all other $j^{\prime} \neq j, \boldsymbol{v}_{i+1}\left(j^{\prime}\right) \geq \boldsymbol{v}_{i}\left(j^{\prime}\right)$.

- if $l=($ if $z, j)$ for some $j=1 \ldots, k$, then $\boldsymbol{v}_{i}(j)=0$ and for all $j^{\prime}=$ $1, \ldots, k, \boldsymbol{v}_{i+1}\left(j^{\prime}\right) \geq \boldsymbol{v}_{i}\left(j^{\prime}\right)$.

Theorem 24 [6, Theorem 2.9] (See also [11, Theorem 6] and [14]) The nonemptiness problem for incrementing counter automata is decidable, but not primitive recursive.

\section{B Register automata}

We are only going to sketch roughly the definition of register automata. Readers interested in its more formal treatment can consult [6, 9]. In essence, $k$ register automaton, or, shortly $k$-RA, is a finite state automaton equipped 
with a header to scan the input and $k$ registers, numbered from 1 to $k$. Each register can store exactly one data value from $\mathfrak{D}$. The automaton is two-way if the header can move to the left or to the right. It is alternating if it is allowed to branch into a finite number of parallel computations.

More formally, a two-way alternating $k$-RA over the label $\Sigma$ is a tuple $\mathcal{A}=\left\langle\Sigma, Q, q_{0}, u_{0}, \mu, F\right\rangle$ where

- $Q_{0}, q_{0} \in Q$ and $F \subseteq Q$ are the finite state of states, the initial state and the set of final states, respectively.

- $u_{0}=a_{1} \cdots a_{k}$ is the initial content of the registers.

- $\mu$ is a set of transitions of the following form.

i) $(q, \sigma) \rightarrow q^{\prime}$ where $a \in\{\triangleleft, \triangleright\}$ and $q, q^{\prime} \in Q$.

That is, if the automaton $\mathcal{A}$ is in state $q$ and the header is currently reading either of the symbols $\triangleleft, \triangleright$, then the automaton can enter the state $q^{\prime}$.

ii) $(q, \sigma, V) \rightarrow q^{\prime}$ where $\sigma \in \Sigma, V \subseteq\{1, \ldots, k\}$ and $q, q^{\prime} \in Q$.

That is, if the automaton $\mathcal{A}$ is in state $q$ and the header is currently reading a position labeled with $\sigma$ and $V$ is the set of all registers containing the current data value, then the automaton can enter the state $q^{\prime}$.

iii) $q \rightarrow\left(q^{\prime}, I\right)$ where $I \subseteq\{1, \ldots, k\}$ and $q, q^{\prime} \in Q$.

That is, if the automaton $\mathcal{A}$ is in state $q$, then the automaton can enter the state $q^{\prime}$ and store the current data value into the registers whose indices belong to $I$.

iv) $q \rightarrow\left(q_{1} \wedge \cdots \wedge q_{i}\right)$ and $q \rightarrow\left(q_{1} \vee \cdots \vee q_{i}\right)$ where $i \geq 1$ and $q, q^{\prime} \in Q$. That is, if the automaton $\mathcal{A}$ is in state $q$, then it can decide to perform conjunctive or disjunctive branching into the states $q_{1}, \ldots, q_{i}$.

v) $q \rightarrow\left(q^{\prime}\right.$, act $)$ where act $\in\{$ left, right $\}$ and $q, q^{\prime} \in Q$.

That is, if the automaton $\mathcal{A}$ is in state $q$, then it can enter the state $q^{\prime}$ and move to the next or the previous word position.

A register automaton is called non deterministic if the branchings of state (in item $(i v)$ ) are all disjunctive. It is called one-way if the header is not allowed to move to the previous word position. 
A configuration $\gamma=\left[j, q, b_{1} \cdots b_{k}\right]$ of the automaton $\mathcal{A}$ consists of the current position of the header in the input word $j$, the state of the automaton $q$ and the content of the registers $b_{1} \cdots b_{k}$. The configuration $\gamma$ is called accepting if the state is a final state in $F$.

From each configuration $\gamma$, the automaton performs legitimate computation according to the transition relation and enters another configuration $\gamma^{\prime}$. If the transition is branching, then it can split into several configurations $\gamma_{1}^{\prime}, \ldots, \gamma_{m}^{\prime}$

Similarly, we can define the notion of leads to acceptance for a configuration $\gamma$ as follows.

- Every accepting configuration leads to acceptance.

- If $\gamma^{\prime}$ is the configuration obtained from $\gamma$ by applying a non-branching transition, then $\gamma$ leads to acceptance if and only if $\gamma^{\prime}$ leads to acceptance.

- If $\gamma_{1}^{\prime}, \ldots, \gamma_{m}^{\prime}$ are the configurations obtained from $\gamma$ by applying a disjunctive branching transition, then $\gamma$ leads to acceptance if and only if at least one of $\gamma_{1}^{\prime}, \ldots, \gamma_{m}^{\prime}$ leads to acceptance.

- If $\gamma_{1}^{\prime}, \ldots, \gamma_{m}^{\prime}$ are the configurations obtained from $\gamma$ by applying a conjunctive branching transition, then $\gamma$ leads to acceptance if and only if all of $\gamma_{1}^{\prime}, \ldots, \gamma_{m}^{\prime}$ lead to acceptance.

An input word $w$ is accepted by $\mathcal{A}$ if the initial configuration leads to acceptance. As usual, $L(\mathcal{A})$ denotes the language accepted by $\mathcal{A}$.

\section{Generalization of Theorem 7}

Let $\mathcal{A}=\left\langle Q, q_{0}, \mu, F\right\rangle$ be a weak $k$-PA. We will show how to construct oneway alternating $(k-1)$-RA $\mathcal{A}^{\prime}$. For our convenience, we assume that $\mathcal{A}$ is deterministic. We also assume that $\mathcal{A}$ behaves as follows.

- For every configuration $\gamma$ of $\mathcal{A}$, there exists a transition in $\mu$ that applies to it.

- Only pebble $k$ can enter a final state and it does so only after it reads the right-end marker $\triangleright$. 
- For every $i=2, \ldots, k$, immediately after pebble $i$ moves right, pebble $i-1$ is placed.

- For every $i=1, \ldots, k-1$, pebble $i$ is lifted only when it reaches the right-end marker $\triangleright$.

- For every $i=1, \ldots, k-1$, immediately after pebble $i$ is lifted, pebble $(i+1)$ moves right.

See Subsection D.1 on how this normalization can be done.

We also assume that the set of states $Q$ is partitioned into $Q_{1} \cup \cdots \cup Q_{k}$ where $Q_{i} \cap Q_{j}$ whenever $i \neq j$ and $Q_{i}$ is the set of states when pebble $i$ is in control.

The automaton $\mathcal{A}^{\prime}=\left\langle Q^{\prime}, q_{0}^{\prime}, u_{0}, \mu^{\prime}, F^{\prime}\right\rangle$ is defined as follows.

- The set of states is $Q^{\prime}=Q \cup Q^{2} \cup Q^{3} \cup \widetilde{Q} \cup \widetilde{Q \times Q}$, where $\widetilde{Q}=\{\tilde{q} \mid q \in Q\}$ and $\widetilde{Q \times Q}=\{\widetilde{(q, p)} \mid p, q \in Q\}$.

- The initial state is $q_{0}^{\prime}=q_{0} \in Q_{k}$.

- The initial assignment is $\#^{k-1}$.

- The set of final states is $F^{\prime}=F \cup\{(q, q): q \in Q\}$.

For our convenience, we number the registers of $\mathcal{A}^{\prime}$ from 2 to $k$, not from 1 to $(k-1)$. The set of transitions $\mu^{\prime}$ consists of the following.

- For $i=k-1, \ldots, 1$, we have the following transitions.

1. For each transition $(i, \sigma, V, q) \rightarrow\left(q^{\prime}\right.$, right $) \in \mu$, there are transitions $((q, p), \sigma, V) \rightarrow\left(q^{\prime}, p\right) \in \mu^{\prime}$ for all $p \in Q_{i+1}$.

2. For each transition $(i, P, V, q) \rightarrow\left(q^{\prime}\right.$, place-pebble $) \in \mu$, there are the following transitions in $\mu^{\prime}$. For every $p \in Q_{i+1}$,

$$
\begin{aligned}
(q, p) & \rightarrow \widetilde{(q, p)},\{i\} \\
(q, p) & \rightarrow \bigvee_{p_{j} \in Q_{i}}\left(q, p_{j}, p\right) \\
\left(q, p_{j}, p\right) & \rightarrow\left(p_{j}, p\right) \wedge\left(q^{\prime}, p_{j}\right) \text { for every } p_{j} \in Q_{i}
\end{aligned}
$$

- For $i=k$, there are the following transitions in $\mu^{\prime}$. 
1. For each transition $(k, \sigma, \emptyset, q) \rightarrow\left(q^{\prime}\right.$, right $) \in \mu$, there is a transition $(q, \sigma, \emptyset) \rightarrow\left(q^{\prime}\right) \in \mu^{\prime}$.

2. For each transition $(k, \sigma, \emptyset, q) \rightarrow\left(q^{\prime}\right.$, place-pebble $) \in \mu$, there are the following transitions in $\mu^{\prime}$.

$$
\begin{aligned}
q & \rightarrow \tilde{q},\{k\} \\
\tilde{q} & \rightarrow \bigvee_{p_{j} \in Q_{k}}\left(q, p_{j}\right) \\
\left(q, p_{j}\right) & \rightarrow p_{j} \wedge\left(q^{\prime}, p_{j}\right) \text { for every } p_{j} \in Q_{k}
\end{aligned}
$$

We can show the following proposition by straightforward induction on $i$.

Proposition 25 Let $w=\left(\begin{array}{c}\sigma_{1} \\ a_{1}\end{array}\right) \cdots\left(\begin{array}{c}\sigma_{n} \\ a_{n}\end{array}\right)$ be a $\Sigma$-data word. For $i=1, \ldots, k-1$, there exists an $i$-run $\left[i, q_{1}, \theta_{1}\right] \vdash^{*}\left[i, q_{2}, \theta_{2}\right]$ of $\mathcal{A}$ on $w$ and $\left[i, q_{2}, \theta_{2}\right] \vdash[i+$ $\left.1, q_{3}, \theta_{3}\right]$ if and only if the configuration $\left[\theta(i),\left(q_{1}, q_{3}\right), u_{2} \cdots u_{k}\right]$ of $\mathcal{A}^{\prime}$ on $w$ leads to acceptance, where $u_{j}=a_{\theta(j)}$, for $j=i+1, \ldots, k$.

Then, by the definition of $\mu^{\prime}$, we can easily deduce the following. For each $\ell=1, \ldots, n$,

$$
\left[k, q_{1}, \theta_{1}\right] \vdash_{\mathcal{A}}\left[k-1, q_{2}, \theta_{2}\right] \vdash_{\mathcal{A}}^{*}\left[k-1, q_{3}, \theta_{3}\right] \vdash_{\mathcal{A}}\left[k, q_{4}, \theta_{4}\right] \vdash_{\mathcal{A}}\left[k, q_{5}, \theta_{5}\right]
$$

is a $k$-run of $\mathcal{A}$ on $w$, where $\theta_{1}(k)=\theta_{2}(k)=\theta_{3}(k)=\theta_{4}(k)=\ell$ and $\theta_{5}(k)=$ $\ell+1$ and $\theta_{2}(k-1)=\ell, \theta_{3}(k-1)=n+1$ if and only if

$$
\begin{aligned}
{\left[\ell, q_{1}, \#^{k-2} a_{\ell-1}\right] } & \vdash\left[\ell, \tilde{q}_{1}, \#^{k-2} a_{\ell}\right] \\
{\left[\ell, \tilde{q}_{1}, \#^{k-2} a_{\ell}\right] } & \vdash\left[\ell,\left(q_{1}, q_{4}\right), \#^{k-2} a_{\ell}\right] \\
{\left[\ell,\left(q_{1}, q_{4}\right), \#^{k-2} a_{\ell}\right] } & \vdash\left[\ell, q_{4}, \#^{k-2} a_{\ell}\right] \\
{\left[\ell,\left(q_{1}, q_{4}\right), \#^{k-2} a_{\ell}\right] } & \vdash\left[\ell,\left(q_{2}, q_{4}\right), \#^{k-2} a_{\ell}\right] \\
{\left[\ell, q_{4}, \#^{k-2} a_{\ell}\right] } & \vdash\left[\ell+1, q_{5}, \#^{k-2} a_{\ell}\right]
\end{aligned}
$$

and the configuration $\left[\ell,\left(q_{2}, q_{4}\right), \#^{k-2} a_{\ell}\right]$ leads to acceptance.

Now, the equivalence between $L(\mathcal{A})$ and $L\left(\mathcal{A}^{\prime}\right)$ follows immediately.

\section{Equivalence between alternating and de- terministic one-way weak $k$-PA}

For every one-way alternating weak $k$-PA, we will construct its equivalent one-way deterministic weak $k$-PA. This is done in two steps. 
1. First, we transform the one-way alternating weak $k$-PA into its equivalent one-way nondeterministic weak $k$-PA.

2. Then, we transform the one-way nondeterministic weak $k$-PA into its equivalent one-way deterministic weak $k$-PA.

We present step 2 first.

\section{D.1 From nondeterministic to deterministic}

We start with the simple case. We will show how to determinize nondeterministic weak 2-PA. The idea can be generalized to arbitrary number of pebbles.

Let $\mathcal{A}=\left\langle Q, q_{0}, F, \mu\right\rangle$ be a nondeterministic weak 2-PA. We start by normalizing the behavior of $\mathcal{A}$ as follows.

N1. For every configuration $\gamma$ of $\mathcal{A}$, there exists a transition in $\mu$ that applies to it.

N2. Only pebble 2 can enter a final state and it does so only after it reads the right-end marker $\triangleright$.

N3. Immediately after pebble 2 moves right, pebble 1 is placed.

N4. Pebble 1 is lifted only when it reaches the right-end marker $\triangleright$.

Such normalization can be done by adding some extra states to $\mathcal{A}$. This normalization N4 is especially important, as it implies that nondeterminism on pebble 1 is now limited only to deciding which state to enter. There is no nondeterminism in choosing which action to take, i.e. either to lift pebble 1 or to keep on moving right.

Next, we note that immediately after pebble 1 is lifted, there can be two choices of actions for pebble 2 :

- to place pebble 1 again; or

- moves pebble 2 to the right.

The following fifth normalization is supposed to handle this situation:

N5. Immediately after pebble 1 is lifted, pebble 2 moves right. 
In other words, while pebble 2 is reading a specific position, pebble 1 makes exactly one pass, from the position of pebble 2 to the right end of the input, instead of making several rounds of passes by placing pebble 1 again immediately after it is lifted. Since there are only finitely many states, there can only be finitely many passes. So, the normalization N4 can be achieved by simultaneously simulating all possible passes in one pass.

With the normalization N1-N5, there is no nondeterminism in choosing which action to take for pebble 2 . The same as for pebble 1 , the nondeterminism for pebble 2 is now limited only in deciding which states to take. This is summed up in the following remark.

Remark 26 For each $i=1,2$, if $(i, P, V, p) \rightarrow\left(q_{1}\right.$, act $\left._{1}\right)$ and $(i, P, V, p) \rightarrow$ $\left(q_{2}\right.$, act $\left._{2}\right)$, then act $_{1}=$ act $_{2}$.

Now that the nondeterminism is reduced to deciding which state to enter, the determinization of $\mathcal{A}$ becomes straightforward. Similar to the classical proof of the equivalence between nondeterministic and deterministic finite state automata, we can take the power set of the states of $\mathcal{A}$ to deterministically simulate $\mathcal{A}$.

Now the normalization steps N1-N5 can be performed similarly for weak $k$-PA $\mathcal{A}$.

$\mathrm{N} 1^{\prime}$. For every configuration $\gamma$ of $\mathcal{A}$, there exists a transition in $\mu$ that applies to it.

$\mathrm{N} 2^{\prime}$. Only pebble $k$ can enter a final state and it does so only after it reads the right-end marker $\triangleright$.

$\mathrm{N} 3^{\prime}$. For each $i=2, \ldots, k$, immediately after pebble $i$ moves right, pebble $(i-1)$ is placed.

$\mathrm{N} 4^{\prime}$. For each $i=1, \ldots, k-1$, pebble $i$ is lifted only when it reaches the right-end marker $\triangleright$.

N5'. For each $i=1, \ldots, k-1$, Immediately after pebble $i$ is lifted, pebble $i+1$ moves right.

Such normalization results in reducing the nondeterminism to deciding which state to enter. Then, the determinization of $\mathcal{A}$ can be done just like in the classical case as in the case of weak 2-PA described above.

The following are the details of the determinization of $\mathcal{A}=\left\langle\Sigma, Q, q_{0}, F\right\rangle$. 
Let $P, V \subseteq\{1, \ldots, k\}$. For a subset $S \subseteq Q$, we define the following:

$E_{i, P, V}(S)=\left\{q: \exists q^{\prime} \in S\right.$ such that $\left(i, P, V, q^{\prime}\right) \rightarrow(q$, act $\left.) \in \mu\right\} ;$

$A_{i, P, V}(S)=$ act where $(i, P, V, q) \rightarrow\left(q^{\prime}\right.$, act $)$ for some $q \in S$ and $q^{\prime} \in Q$.

By Remark 26 above, $A_{i, P, V}(S)$ is well defined. Furthermore, $E_{i, P, V}$ is monotone, that is, if $S \subseteq S^{\prime}$, then $E_{i, P, V}(S) \subseteq E_{i, P, V}\left(S^{\prime}\right)$.

Now we present the construction of a deterministic, weak $k$-pebble automaton $\mathcal{A}^{\prime}$ equivalent to $\mathcal{A}$. Let $\mathcal{A}^{\prime}=\left\langle Q^{\prime}, q_{0}^{\prime}, F^{\prime}, \mu^{\prime}\right\rangle$ where

- $Q^{\prime}=2^{Q}$

- $q_{0}^{\prime}=\left\{q_{0}\right\}$

- $F^{\prime}=\{S \subseteq Q: S \cap F \neq \emptyset\}$

- $\mu^{\prime}$ consists of the following transitions. For each $i \in\{1, \ldots, k\}, P, V \subseteq$ $\{i+1, \ldots, k\}$ and $S \subseteq Q$,

$$
(i, P, V, S) \rightarrow\left(E_{i, P, V}(S), A_{i, P, V}(S)\right) \in \mu^{\prime} .
$$

Recall that an $i$-run is a run from an $i$-configuration to an $i$-configuration in which pebble $i+1$ is never lifted. We are going to use the following Claim 1 to prove that $L\left(\mathcal{A}^{\prime}\right)=L(\mathcal{A})$.

Claim 1 Let $i=1, \ldots, k$. Let $w \in \Sigma^{*}$ and $\theta_{1}, \theta_{2}$ be pebble assignments on $w$.

1. For every set of states $S_{1}, S_{2} \subseteq Q$, if $\left[i, S_{1}, \theta_{1}\right] \vdash^{*}\left[i, S_{2}, \theta_{2}\right]$ is an i-run of $\mathcal{A}^{\prime}$, then

$$
\bigcup_{q_{1} \in S_{1}}\left\{\gamma:\left[i, q_{1}, \theta_{1}\right] \vdash_{\mathcal{A}}^{*} \gamma \text { is an i-run }\right\}=\left\{\left[i, q_{2}, \theta_{2}\right]: q_{2} \in S_{2}\right\} .
$$

2. For every state $q_{1}, q_{2} \subseteq Q$, if $\left[i, q_{1}, \theta_{1}\right] \vdash^{*}\left[i, q_{2}, \theta_{2}\right]$ is an $i$-run of $\mathcal{A}$, then for all $S_{1} \subseteq Q$ such that $q_{1} \in S_{1}$, there exists $S_{2} \subseteq Q$ such that $q_{2} \in S_{2}$ and $\left[i, S_{1}, \theta_{1}\right] \vdash^{*}\left[i, S_{2}, \theta_{2}\right]$ is an $i$-run of $\mathcal{A}^{\prime}$. 
Proof. Let $w, \theta_{1}, \theta_{2}$ be as above. The proof of the claim is by induction on $i$. The base case, $i=1$, is the same as the standard finite state automaton, thus, omitted.

For the induction hypothesis, we assume that the claim is true for the case of $i-1$. To proceed with the induction step, we prove the claim for the case $i$.

We start by proving (1). Let $S_{1}, S_{2} \subseteq Q$. Assume that

$$
\left[i, S_{1}, \theta_{1}\right] \vdash^{*}\left[i, S_{2}, \theta_{2}\right]
$$

By our normalization of $\mathcal{A}$, the resulting automaton $\mathcal{A}^{\prime}$ from our construction is also normalized as the automaton $\mathcal{A}$. Thus, an $i$-run of $\mathcal{A}^{\prime}$ is a repeated sequence of transitions relations of the form:

$$
\left[i, R_{1}, \lambda_{1}\right] \vdash\left[i-1, R_{2}, \lambda_{2}\right] \vdash^{*}\left[i-1, R_{3}, \lambda_{3}\right] \vdash\left[i, R_{4}, \lambda_{4}\right] \vdash\left[i, R_{5}, \lambda_{5}\right],
$$

where

a) some transition $\left(i, P_{1}, V_{1}, R_{1}\right) \rightarrow\left(R_{2}\right.$, place-pebble $) \in \mu^{\prime}$ is applied to obtain the transition relation $\left[i, R_{1}, \lambda_{1}\right] \vdash\left[i-1, R_{2}, \lambda_{2}\right]$,

b) the transition relation $\left[i-1, R_{2}, \lambda_{2}\right] \vdash^{*}\left[i-1, R_{3}, \lambda_{3}\right]$ is an $(i-1)$-run of $\mathcal{A}^{\prime}$,

c) some transition $\left(i, P_{2}, V_{2}, R_{3}\right) \rightarrow\left(R_{4}\right.$, lift-pebble $) \in \mu^{\prime}$ is applied to obtain the transition relation $\left[i, R_{3}, \lambda_{3}\right] \vdash\left[i, R_{4}, \lambda_{4}\right]$,

d) some transition $\left(i, P_{3}, V_{3}, R_{4}\right) \rightarrow\left(R_{5}\right.$, right $) \in \mu^{\prime}$ is applied to obtain the transition relation $\left[i, R_{4}, \lambda_{4}\right] \vdash\left[i, R_{5}, \lambda_{5}\right]$.

Thus, to prove part (1), it suffices to prove the following four equations.

$$
\begin{aligned}
\bigcup_{p_{1} \in R_{1}}\left\{\gamma:\left[i, p_{1}, \lambda_{1}\right] \vdash_{\mathcal{A}} \gamma\right\} & =\left\{\left[i-1, p_{2}, \lambda_{2}\right]: p_{2} \in R_{2}\right\} \\
\bigcup_{p_{2} \in R_{2}}\left\{\gamma:\left[i-1, p_{2}, \lambda_{2}\right] \vdash_{\mathcal{A}}^{*} \gamma \text { is an }(i-1) \text {-run }\right\} & =\left\{\left[i-1, p_{3}, \lambda_{3}\right]: p_{3} \in R_{3}\right\} \\
\bigcup_{p_{3} \in R_{3}}\left\{\gamma:\left[i-1, p_{3}, \lambda_{3}\right] \vdash_{\mathcal{A}} \gamma\right\} & =\left\{\left[i, p_{4}, \lambda_{4}\right]: p_{4} \in R_{4}\right\} \\
\bigcup_{p_{4} \in R_{4}}\left\{\gamma:\left[i, p_{4}, \lambda_{4}\right] \vdash_{\mathcal{A}} \gamma\right\} & =\left\{\left[i, p_{5}, \lambda_{5}\right]: p_{5} \in R_{5}\right\}
\end{aligned}
$$


Proof of ([6): By item (a) above, there is a transition $\left(i, P_{1}, V_{1}, R_{1}\right) \rightarrow$ $\left(R_{2}\right.$, place-pebble $) \in \mu^{\prime}$. By the construction of $\mu^{\prime}$ that $R_{2}=E_{i, P_{1}, V_{1}}\left(R_{1}\right)$, we immediately have Equation 6.

Proof of (7): By item (b) above, $\left[i-1, R_{2}, \lambda_{2}\right] \vdash^{*}\left[i-1, R_{3}, \lambda_{3}\right]$ is an $(i-1)$-run of $\mathcal{A}^{\prime}$. Equation 7 follows from the induction hypothesis.

Proof of (8): By item (c) above, there is a transition $\left(i, P_{2}, V_{2}, R_{3}\right) \rightarrow$ $\left(R_{4}\right.$, lift-pebble $) \in \mu^{\prime}$. By the construction of $\mu^{\prime}$ that $R_{4}=E_{i, P_{2}, V_{2}}\left(R_{3}\right)$, we immediately have Equation 8.

Proof of (9): By item (d) above, there is a transition $\left(i, P_{3}, V_{3}, R_{4}\right) \rightarrow$ $\left(R_{5}\right.$, right $) \in \mu^{\prime}$. By the construction of $\mu^{\prime}$ that $R_{5}=E_{i, P_{3}, V_{3}}\left(R_{4}\right)$, we immediately have Equation 9 .

The proof of part (2) of our claim is very similar to the one of part (1). For completeness, we present it here. Let $q_{1}, q_{2} \in Q$ and

$$
\left[i, q_{1}, \theta_{1}\right] \vdash^{*}\left[i, q_{2}, \theta_{2}\right]
$$

be an $i$-run of $\mathcal{A}$.

By our normalization of $\mathcal{A}$, an $i$-run of $\mathcal{A}^{\prime}$ is a repeated sequence of transitions relations of the form:

$$
\left[i, p_{1}, \lambda_{1}\right] \vdash\left[i-1, p_{2}, \lambda_{2}\right] \vdash^{*}\left[i-1, p_{3}, \lambda_{3}\right] \vdash\left[i, p_{4}, \lambda_{4}\right] \vdash\left[i, p_{5}, \lambda_{5}\right]
$$

where

a) some transition $\left(i, P_{1}, V_{1}, p_{1}\right) \rightarrow\left(p_{2}\right.$, place-pebble $) \in \mu$ is applied to obtain the transition relation $\left[i, p_{1}, \lambda_{1}\right] \vdash\left[i-1, p_{2}, \lambda_{2}\right]$,

b) the transition relation $\left[i-1, p_{2}, \lambda_{2}\right] \vdash^{*}\left[i-1, p_{3}, \lambda_{3}\right]$ is an $(i-1)$-run of $\mathcal{A}^{\prime}$

c) some transition $\left(i, P_{2}, V_{2}, p_{3}\right) \rightarrow\left(p_{4}\right.$, lift-pebble $) \in \mu^{\prime}$ is applied to obtain the transition relation $\left[i-1, p_{3}, \lambda_{3}\right] \vdash\left[i-1, p_{4}, \lambda_{4}\right]$,

d) some transition $\left(i, P_{3}, V_{3}, p_{4}\right) \rightarrow\left(p_{5}\right.$, right $) \in \mu^{\prime}$ is applied to obtain the transition relation $\left[i, p_{4}, \lambda_{4}\right] \vdash\left[i, p_{5}, \lambda_{5}\right]$.

The proof of part (2) follows from the following. 
- For all $R_{1} \subseteq Q$ such that $p_{1} \in R_{1}$, there exists $R_{2} \subseteq Q$ such that $p_{2} \in R_{2}$ and $\left[i, R_{1}, \lambda_{1}\right] \vdash_{\mathcal{A}^{\prime}}\left[i-1, R_{2}, \lambda_{2}\right]$.

This immediately follows from the fact that $p_{2} \in E_{i, P_{1}, V_{1}}\left(R_{1}\right)$ and $\left(i, P_{1}, V_{1}, R_{1}\right) \rightarrow\left(E_{i, P_{1}, V_{1}}\left(R_{1}\right)\right.$, place-pebble $) \in \mu^{\prime}$.

- For all $R_{2} \subseteq Q$ such that $p_{2} \in R_{2}$, there exists $R_{3} \subseteq Q$ such that $p_{3} \in R_{3}$ and $\left[i-1, R_{2}, \lambda_{2}\right] \vdash_{\mathcal{A}^{\prime}}^{*}\left[i-1, R_{3}, \lambda_{3}\right]$ is an $(i-1)$-run.

This follows from the induction hypothesis.

- For all $R_{3} \subseteq Q$ such that $p_{3} \in R_{3}$, there exists $R_{4} \subseteq Q$ such that $p_{4} \in R_{4}$ and $\left[i-1, R_{3}, \lambda_{3}\right] \vdash_{\mathcal{A}^{\prime}}\left[i, R_{4}, \lambda_{4}\right]$.

This immediately follows from the fact that $p_{3} \in E_{i, P_{2}, V_{2}}\left(R_{3}\right)$ and $\left(i, P_{2}, V_{2}, R_{3}\right) \rightarrow\left(E_{i, P_{2}, V_{2}}\left(R_{3}\right)\right.$, lift-pebble $) \in \mu^{\prime}$.

- For all $R_{4} \subseteq Q$ such that $p_{4} \in R_{4}$, there exists $R_{5} \subseteq Q$ such that $p_{5} \in R_{5}$ and $\left[i, R_{4}, \lambda_{4}\right] \vdash_{\mathcal{A}^{\prime}}\left[i, R_{5}, \lambda_{5}\right]$.

This immediately follows from the fact that $p_{4} \in E_{i, P_{3}, V_{3}}\left(R_{4}\right)$ and $\left(i, P_{3}, V_{3}, R_{4}\right) \rightarrow\left(E_{i, P_{3}, V_{3}}\left(R_{4}\right)\right.$, right $) \in \mu^{\prime}$.

\section{D.2 From alternating to nondeterministic}

Let $\mathcal{A}=\left\langle\Sigma, Q, q_{0}, \mu, F, U\right\rangle$ be one-way alternating weak $k$-PA. Adding some extra states, we can normalize $\mathcal{A}$ as follows.

- For every $p \in U$, if $(i, \sigma, V, p) \rightarrow(q$, act $) \in \mu$, then act $=$ stay.

- Every pebble can be lifted only after it reads the right-end marker $\triangleright$.

- Only pebble $k$ can enter a final state and it does so only after it reads the right-end marker $\triangleright$.

We assume that $Q$ is partitioned into $Q_{1} \cup \cdots \cup Q_{k}$ where $Q_{i}$ is the set of states, where pebble $i$ is the head pebble, for each $i=1, \ldots, k$. We can further partition each $Q_{i}$ into four sets of states: $Q_{i, \text { stay }}, Q_{i, \text { right }}, Q_{i \text {,place }}$, $Q_{i, \text { lift }}$ such that for every $i, \sigma, V, q$ and $p$,

- if $q \in Q_{i, \text { stay }}$ and $(i, \sigma, V, q) \rightarrow(p$, act $) \in \mu$, then act = stay;

- if $q \in Q_{i, \text { right }}$ and $(i, \sigma, V, q) \rightarrow(p$, act $) \in \mu$, then act $=$ right; 
- if $q \in Q_{i, \text { place }}$ and $(i, \sigma, V, q) \rightarrow(p$, act $) \in \mu$, then act $=$ place-pebble;

- if $q \in Q_{i, 1 \text { ift }}$ and $(i, \sigma, V, q) \rightarrow(p$, act $) \in \mu$, then act $=$ lift-pebble.

Now we define a nondeterministic weak $k$-PA $\mathcal{A}^{\prime}=\left\langle\Sigma, Q^{\prime}, q_{0}^{\prime}, \mu^{\prime}, F^{\prime}\right\rangle$, where

- $Q^{\prime}$ consists of states of the form $\left(S_{k}, S_{k-1} \ldots, S_{k-j}\right) \in 2^{Q_{k}} \times 2^{Q_{k-1}} \times$ $2^{Q_{k-j}}$, where $0 \leq j \leq k-1$;

- $q_{0}^{\prime}=\left\{q_{0}\right\}$

- $F^{\prime}=2^{F}-\{\emptyset\}$.

The set $\mu^{\prime}$ contains the following transitions. For every $i=1,2, \ldots, k$, for every $V \subseteq\{i+1, \ldots, k\}$, for every $\left(S_{k}, S_{k-1}, \ldots, S_{i}\right) \in Q^{\prime}$, for every $\sigma \in \Sigma$, we have the following transitions.

- If $S_{i}$ contains a state $q \in U$, then $\left(i, \sigma, V,\left(S_{k}, S_{k-1}, \ldots, S_{i}\right)\right) \rightarrow\left(\left(S_{k}, S_{k-1}, \ldots,\left(S_{i}-\{q\}\right) \cup U_{q}\right)\right.$, stay $) \in \mu^{\prime}$ where $U_{q}=\{p \mid(i, \sigma, V, q) \rightarrow(p$, stay $) \in \mu\}$.

- If $S_{i}$ contains a state $q \in Q_{i, \text { stay }}$ and $S \cap U=\emptyset$, then $\left(i, \sigma, V,\left(S_{k}, S_{k-1}, \ldots, S_{i}\right)\right) \rightarrow\left(\left(S_{k}, S_{k-1}, \ldots,\left(S_{i}-\{q\}\right) \cup N_{q}\right)\right.$, stay $) \in \mu^{\prime}$ where $N_{q} \subseteq\{p \mid(i, \sigma, V, q) \rightarrow(p$, stay $) \in \mu\}$.

- If $S_{i}$ contains a state $q \in Q_{i \text {,place }}$ and $S_{i} \cap Q_{i, \text { stay }}=\emptyset$, then $\left(i, \sigma, V,\left(S_{k}, S_{k-1}, \ldots, S_{i}\right)\right) \rightarrow\left(\left(S_{k}, S_{k-1}, \ldots, S_{i}-\{q\},\{p\}\right)\right.$, place-pebble $) \in \mu^{\prime}$ where $(i, \sigma, V, q) \rightarrow(p$, place-pebble $) \in \mu$.

- If $S_{i} \subseteq Q_{i, \text { right }}$, then

$$
\left(i, \sigma, V,\left(S_{k}, S_{k-1}, \ldots, S_{i}\right)\right) \rightarrow\left(\left(S_{k}, S_{k-1}, \ldots, S_{i}^{\prime}\right), \text { right }\right) \in \mu^{\prime}
$$

where $S_{i}^{\prime}=\left\{p \mid(i, \sigma, V, q) \rightarrow(p\right.$, right $) \in \mu$ and $\left.q \in S \cap Q_{i}\right\}$.

- If $S_{i} \subseteq Q_{i, 1 \text { ift }}$, then $\left(i, \triangleright, V,\left(S_{k}, S_{k-1}, \ldots, S_{i+1}, S_{i}\right)\right) \rightarrow\left(\left(S_{k}, S_{k-1}, \ldots, S_{i+1} \cup R\right)\right.$, lift-pebble $) \in \mu^{\prime}$ where $R=\left\{p \mid(i, \sigma, V, q) \rightarrow(p\right.$, lift-pebble $) \in \mu$ and $\left.q \in S_{i}\right\}$. 
The proof that $L(\mathcal{A})=L\left(\mathcal{A}^{\prime}\right)$ is pretty much similar to the one in the previous subsection.

Let $S_{k}, S_{k-1}, \ldots, S_{i}$ and $\theta$ such that

$$
\left[k,\left\{q_{0}\right\}, \theta_{0}\right] \vdash_{\mathcal{A}^{\prime}}^{*}\left[i,\left(S_{k}, S_{k-1}, \ldots, S_{i}\right), \theta\right] .
$$

For each $j=k, k-1, \ldots, i$, we define $\theta_{j}$ to be $\theta$ restricted to the domain $\{k, k-1, \ldots, j\}$. Then, by straightforward induction on $i$, we can show that

$$
\left[k, q_{0}, \theta_{0}\right] \vdash_{\mathcal{A}}^{*}\left[j, q, \theta_{j}\right] \text {, for each } j=k, k-1, \ldots, i .
$$

Now we show the converse direction. Let $\theta$ be an assignment for pebbles $k, k-1, \ldots, i$ and we denote by $\theta_{j}$ the pebble assignment $\theta$ restricted to the domain $\{k, k-1, \ldots, j\}$ when $j \geq i$. Let $S_{k}, S_{k-1}, \ldots, S_{i}$ be subsets of $Q_{k}, Q_{k-1}, \ldots, Q_{i}$, respectively, and let $p_{k}, p_{k-1}, p_{i+1}$ be an element of $Q_{k}, Q_{k-1}, \ldots, Q_{i}$ such that for each $j=k, k-1, \ldots, i$,

- $p_{j} \notin S_{j}$;

- $\left[k, q_{0}, \theta_{0}\right] \vdash_{\mathcal{A}}^{*}\left[j, q, \theta_{j}\right]$, for each $q \in S_{j}$.

Then, by straightforward induction on $i$, we can show that

$$
\left[k,\left\{q_{0}\right\}, \theta_{0}\right] \vdash_{\mathcal{A}^{\prime}}^{*}\left[i,\left(S_{k}, S_{k-1}, \ldots, S_{i}\right), \theta\right] .
$$

